\title{
Efficient Synthesis and Versatile Reactivity of Porphyrinyl Grignard Reagents
}

\section{AUTHOR(S):}

Fujimoto, Keisuke; Yorimitsu, Hideki; Osuka, Atsuhiro

\section{CITATION:}

Fujimoto, Keisuke ...[et al]. Efficient Synthesis and Versatile Reactivity of Porphyrinyl Grignard Reagents. European Journal of Organic Chemistry 2014, 2014(20): 4327-4334

\section{ISSUE DATE:}

2014-05-22

URL:

http://hdl.handle.net/2433/200176

\section{RIGHT:}

This is the peer reviewed version of the following article: Fujimoto, K., Yorimitsu, H. and Osuka, A. (2014), Efficient Synthesis and Versatile Reactivity of Porphyrinyl Grignard Reagents. Eur. J. Org. Chem., 2014: 4327-4334, which has been published in final form at http://dx.doi.org/10.1002/ejoc.201402391. This article may be used for non-commercial purposes in accordance with Wiley Terms and Conditions for Self-Archiving.; この論文は出版社版でありません。引用 の際には出版社版をご確認ご利用ください。; This is not the published version. Please cite only the published version. 


\title{
Efficient Synthesis and Versatile Reactivity of Porphyrinyl Grignard Reagents**
}

\author{
Keisuke Fujimoto, ${ }^{[a]}$ Hideki Yorimitsu, ${ }^{[a, b]}$ and Atsuhiro Osuka*[a]
}

Keywords: porphyrin / iodine-magnesium exchange / Grignard reagent / transmetalation

\begin{abstract}
Iodine-magnesium exchange between iodoporphyrins and $i$ PrMgCl-LiCl successfully proceeded without decomposition of the porphyrin core. The resulting porphyrinyl Grignard reagents are nucleophilic enough to react with various carbonyl compounds, such as aldehyde, ketone, and amide. Furthermore, the porphyrinyl

[a] Department of Chemistry, Graduate School of Science, Kyoto University Sakyo-ku Kyoto 606-8502 (Japan)

E-mail: yori@kuchem.kyoto-u.ac.jp; osuka@kuchem.kyoto-u.ac.jp

[b] ACT-C, Japan Science and Technology Agency (Japan)

[**] The work was supported by Grants-in-Aid from MEXT (Nos.: 24106721 "Reaction Integration" and 25107002 "Science of Atomic Layers”) and from JSPS (Nos.: 25220802 (Scientific Research (S)), 24685007 (Young Scientists (A)), 23655037 and 26620081 (Exploratory Research)).

Supporting information for this article is available on the WWW under http://dx.doi.org/10.1002/ejoc.xxxxxxxxx.
\end{abstract}

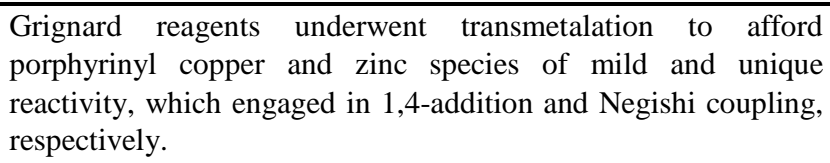
reactivity, which engaged in 1,4-addition and Negishi coupling, respectively.

\section{Introduction}

Porphyrins are an important class of heteroaromatic compounds that play a wide variety of roles in nature such as oxygen transport and photosynthesis. ${ }^{[1]}$ Significant attention has been paid to development of new porphyrins that exhibit interesting and useful properties in catalysis, biological applications, and material sciences. Peripheral functionalizations of porphyrin cores definitely represent an effective process to synthesize porphyrins that have altered properties.

Metalation of the periphery of porphyrins is regarded to be a key step for peripheral functionalization since the resulting carbonmetal bond is reactive to experience various transformations. ${ }^{[2]}$ Direct mercuration is historically important as the first peripheral metalation. ${ }^{[3]}$ Although the resulting carbon-mercury bonds were usefully convertible, the toxicity of mercury would impede the practical applications. In contrast, borylated porphyrins are easily accessible and safely underwent useful transformations, ${ }^{[4]}$ such as Suzuki-Miyaura cross-coupling, oxidative hydroxylation, and halogenation. ${ }^{[5]}$

Considering the importance of these borylated porphyrins, we expected that peripherally magnesiated porphyrins should also be fascinating synthetic intermediates because of their higher nucleophilicity to achieve a wider variety of efficient bond-forming processes. ${ }^{[6]}$ However, synthesis and reactions of magnesiated porphyrins have been still unexplored. Chen et al. reported the only one example of generation of a porphyrinyl Grignard reagent from meso-bromoporphyrin. ${ }^{[7]}$ However, commercially available magnesium turnings did not serve, and preparation of active Rieke magnesium in situ from $\mathrm{MgCl}_{2}, \mathrm{KI}$, and extremely reactive metallic potassium was indispensable. The Grignard reagent reacted with aromatic aldehydes in only low yields and with ketones to anomalously form $\alpha$-porphyrinylated ketones, the scope of electrophiles thus being extremely limited and unusual. These results indicate that the formation of the Grignard reagent is inefficient and accompanied by side reactions. In addition, the reactions should be performed in a Barbier fashion to avoid decomposition of the Grignard reagent. We thus assumed that the efficient generation of the porphyrinyl Grignard reagent was difficult since a porphyrin skeleton is susceptible to nucleophilic attack, ${ }^{[8]}$ single electron transfer, ${ }^{[9]}$ and reductive demetalation ${ }^{[10]}$ under Chen's conditions.

Preparation of functionalized Grignard reagents is rather difficult since the insertion of magnesium metal to a carbon-halogen bond does not work under cryogenic conditions and many functional groups are incompatible under noncryogenic conditions. In 2004, Knochel et al. developed $i \mathrm{PrMgCl} \cdot \mathrm{LiCl}$ as a powerful tool for smooth halogen-magnesium exchange. ${ }^{[11]}$ This breakthrough has realized preparation of a variety of functionalized aryl and heteroaryl Grignard reagents at low temperatures, thereby considerably advancing organic synthesis. We envisioned that porphyrinyl Grignard reagents could be efficiently synthesized at a low temperature through iodine-magnesium exchange with $i \mathrm{PrMgCl} \cdot \mathrm{LiCl}$. This was indeed the case and here we wish to report the first efficient synthesis of porphyrinyl Grignard reagents and their versatile reactivity.

\section{Results and Discussion}

Firstly, we aimed to identify formation of porphyrinyl Grignard reagent $2 \mathrm{Ni}$ prepared through the iodine-magnesium exchange reaction of $\mathrm{Ni}^{\mathrm{II}} \beta$-iodoporphyrin $\mathbf{1} \mathbf{N i}^{[5 \mathrm{Il}}$ (Table $1, \mathrm{Ar}=3,5$-di-tertbutylphenyl and $\boldsymbol{M g}$ that is located at the periphery denotes $\mathrm{MgCl} \cdot \mathrm{LiCl}$ throughout the manuscript.). After treatment of $\mathbf{1 N i}$ with $i \mathrm{PrMgCl} \cdot \mathrm{LiCl}$ in $\mathrm{THF}$ at $-40^{\circ} \mathrm{C}$ for $2 \mathrm{~h}, \mathrm{D}_{2} \mathrm{O}$ was added to the resulting reaction mixture to afford $\beta$-deuterioporphyrin $3 \mathbf{N i}$ in $95 \%$ yield. This result suggests that the iodine-magnesium exchange reaction provided $\mathbf{2 N i}$ without any significant side reactions. Indeed, $\mathbf{2 N i}$ showed typical behavior in the reactions with carbonyl compounds, such as benzaldehyde, cyclohexanone, and dimethylformamide (DMF) to give $\mathbf{4} \mathbf{N i}, \mathbf{5 N i}$, and $\mathbf{6} \mathbf{N i}^{[12]}$ in $78 \%, 71 \%$, and $70 \%$ yields, respectively. Iodine-magnesium 
exchange reaction of the zinc analogue $1 \mathbf{Z n}$ was carried out at a lower temperature because zinc porphyrins were more labile under the reaction conditions. The formation of $\mathrm{Zn}^{\mathrm{II}}$ porphyrinyl Grignard reagent $\mathbf{2 Z n}$ was also confirmed by the reaction with $\mathrm{D}_{2} \mathrm{O}$ to give $\mathbf{3 Z n}$ in $90 \%$ yield. Nucleophilic addition of $\mathbf{2 Z n}$ with cyclohexanone also took place cleanly to provide $5 \mathbf{Z n}$ in $68 \%$ yield.

Table 1. Preparations and reactions of $\beta$-magnesiated porphyrins 2M
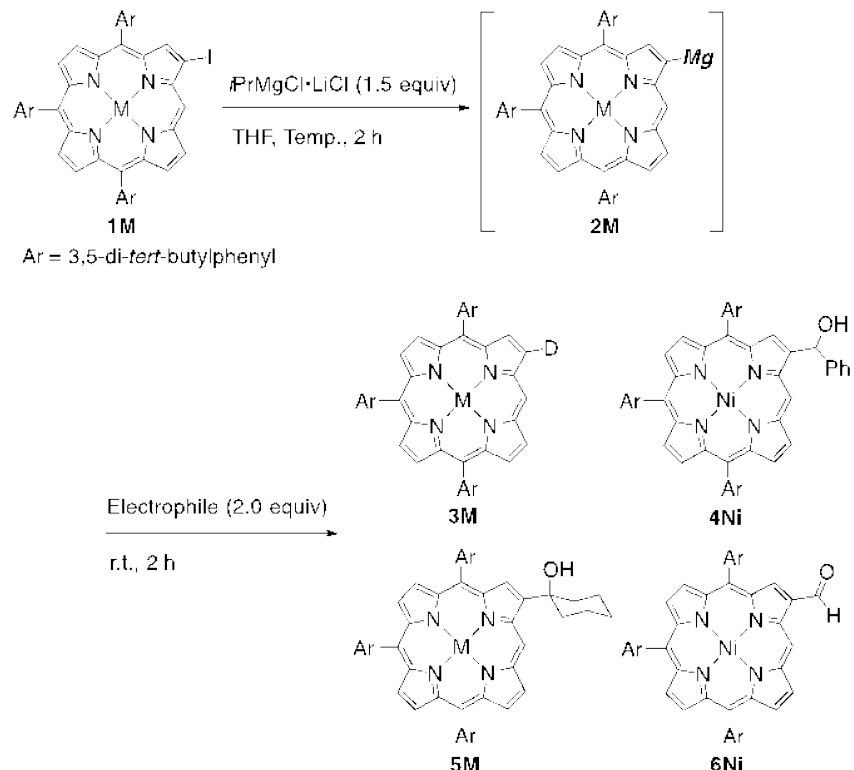

\begin{tabular}{llllll}
\hline Entry & Substrate & Temp. $\left[^{\circ} \mathrm{C}\right]$ & Electrophile & Product & $\begin{array}{l}\text { Yield } \\
{[\%]}\end{array}$ \\
\hline 1 & $\mathbf{1 N i}$ & -40 & $\mathrm{D}_{2} \mathrm{O}$ & $\mathbf{3 N i}$ & $95^{[a]}$ \\
2 & $\mathbf{1 N i}$ & -40 & PhCHO & $\mathbf{4 N i}$ & 78 \\
3 & $\mathbf{1 N i}$ & -40 & cyclohexanone & $\mathbf{5 N i}$ & 70 \\
4 & $\mathbf{1 N i}$ & -40 & DMF & $\mathbf{6 N i}$ & 71 \\
5 & $\mathbf{1 Z n}$ & -80 & $\mathrm{D}_{2} \mathrm{O}$ & $\mathbf{3 Z n}$ & $90^{[a]}$ \\
6 & $\mathbf{1 Z n}$ & -80 & cyclohexanone & $\mathbf{5 Z n}$ & 68 \\
\hline
\end{tabular}

[a] With an excess amount of $\mathrm{D}_{2} \mathrm{O}$ for $5 \mathrm{~min}$.

Scheme 1. Dimagnesiation of $\beta, \beta$ '-diiodoporphyrin $7 \mathbf{N i}$
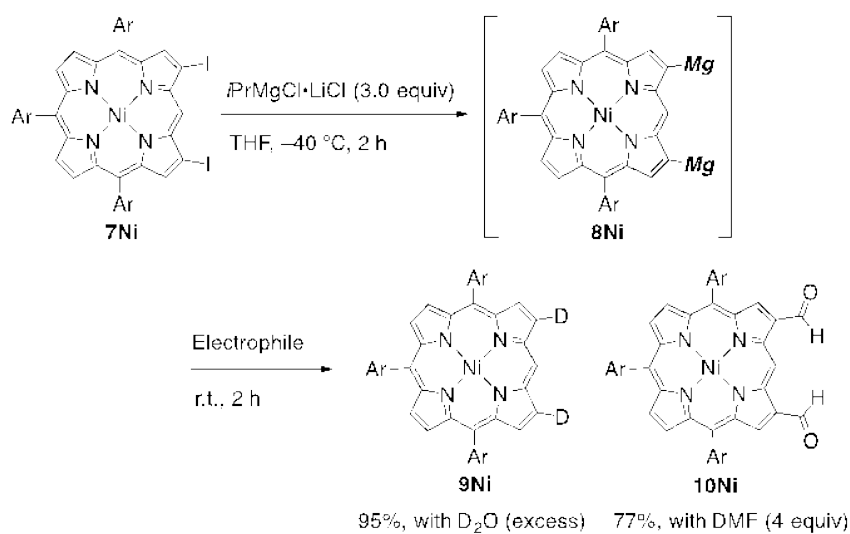

We also attempted two-fold iodine-magnesium exchange of $\mathrm{Ni}^{\mathrm{II}}$ $\beta, \beta$ '-diiodoporphyrin $7 \mathbf{N i}^{[5]]}$ with $i \mathrm{PrMgCl} \cdot \mathrm{LiCl}$ in $\mathrm{THF}$ at $-40{ }^{\circ} \mathrm{C}$ (Scheme 1). The iodine-magnesium exchange was successful, and the resulting dimagnesiated complex $\mathbf{8 N i}$ was trapped with $\mathrm{D}_{2} \mathrm{O}$ or DMF to furnish $\beta, \beta$ '-dideuterio- or diformylporphyrin $\mathbf{9 N i}$ or $10 \mathrm{Ni}$ in $95 \%$ or $77 \%$ yields, respectively.
Encouraged by the success in the reactions of $\beta$-iodoporphyrins, we next tried to apply these procedures to meso-iodoporphyrins $\mathbf{1 1}^{\text {[13] }}$ (Table 2). Similar deuterium labelling experiments strongly suggest quantitative formation of the corresponding Grignard reagent $\mathbf{1 2 M}$ via iodine-magnesium exchange (entries 1 and 5). meso-Magnesiated porphyrin 12Ni also reacted with benzaldehyde to give $14 \mathrm{Ni}$ in a reasonable yield of $68 \%$. Unfortunately, the reactions with DMF required long times and furnished $\mathbf{1 6 M}$ in moderate yields because of the low nucleophilicity of the sterically hindered meso-carbon. The reactions with cyclohexanone provided $\mathbf{1 5} \mathbf{M}^{[12]}$ in low yields due to competitive protonation of $\mathbf{1 2 M}$ with the $\alpha$-protons of cyclohexanone.

Table 2. Preparations and reactions of meso-magnesiated porphyrins $12 \mathbf{M}$

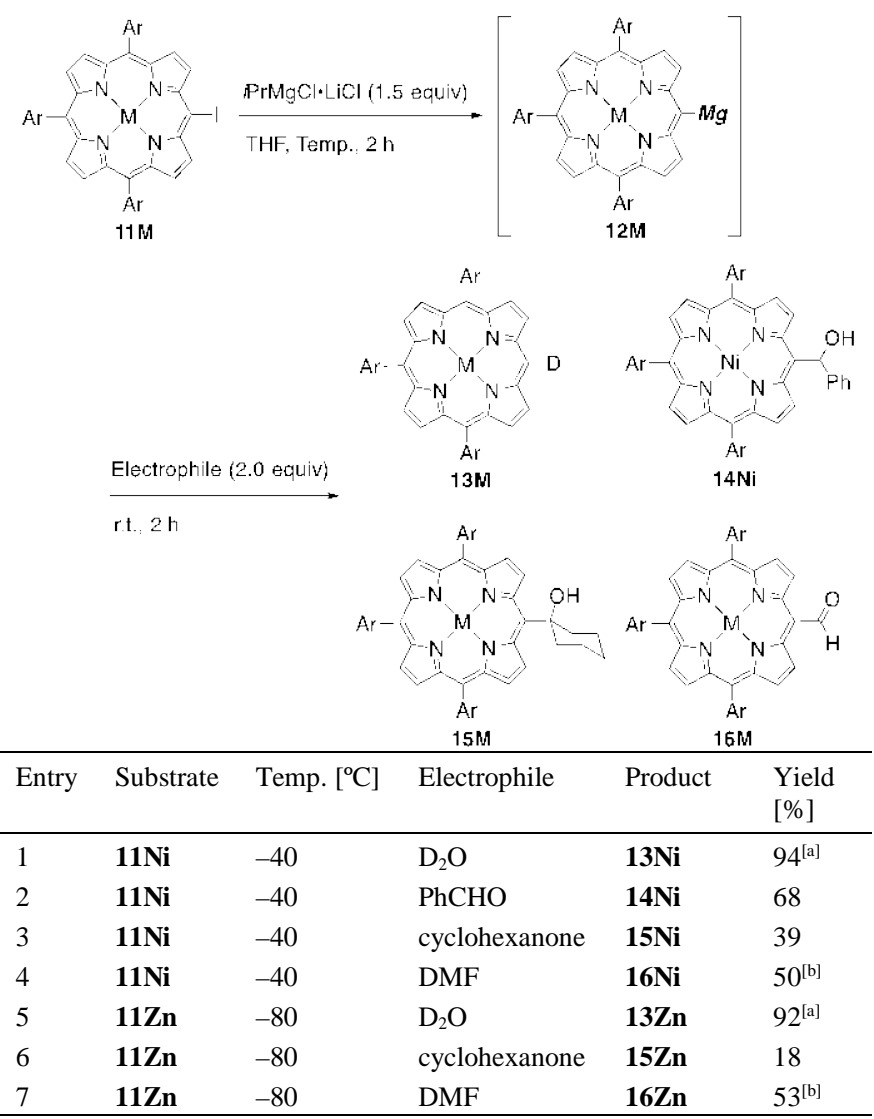

[a] With an excess amount of $\mathrm{D}_{2} \mathrm{O}$ for 5 min. [b] For $24 \mathrm{~h}$.

We then envisioned that the utility of the porphyrinyl Grignard reagents could be extended through transmetalation with other metal salts. Indeed, porphyrinyl copper was generated from the corresponding porphyrinyl magnesium and exhibited desired reactivities (Table 3 ). In the presence of a catalytic amount of $\mathrm{CuCN} \cdot 2 \mathrm{LiCl},{ }^{[11 a]}$ porphyrinyl Grignard reagents $2 \mathrm{Ni}$ and $\mathbf{8 N i}$ reacted with 2-naphthoyl chloride to give $\beta$-(2naphthoyl)porphyrins $\mathbf{1 7} \mathbf{N i}$ and $\mathbf{2 0 N i}{ }^{[12]}$ in $72 \%$ and $62 \%$ yields, respectively. An $\mathrm{S}_{\mathrm{N}} 2$ ' reaction with allyl bromide also proceeded to yield $\beta$-allylporphyrin $\mathbf{1 8 N i}$ efficiently. In the presence of chlorotrimethylsilane, ${ }^{[14]}$ 1,4-addition to 2-cyclohexen-1-one occurred to provide desired adduct $19 \mathrm{Ni}$ in $68 \%$ yield. On the other hand, the reaction of porphyrinyl magnesium 2Ni with 2cyclohexen-1-one without $\mathrm{CuCN} \cdot 2 \mathrm{LiCl}$ gave a rather complicated and inseparable mixture. APCI-TOF MS analysis of the mixture tentatively implied that the mixture included not only $\beta$ unsubstituted porphyrin and 19Ni but also considerable amounts of 
$\beta$-phenylporphyrin and $\beta$-(1,3-cyclohexadienyl)porphyrin, which would result from 1,2-addition to 2-cyclohexen-1-one.

Table 3. Reactions of porphyrinyl copper

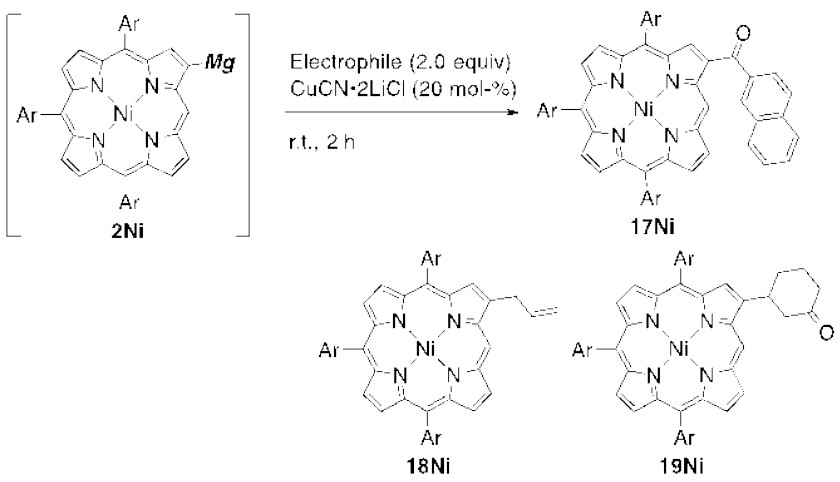

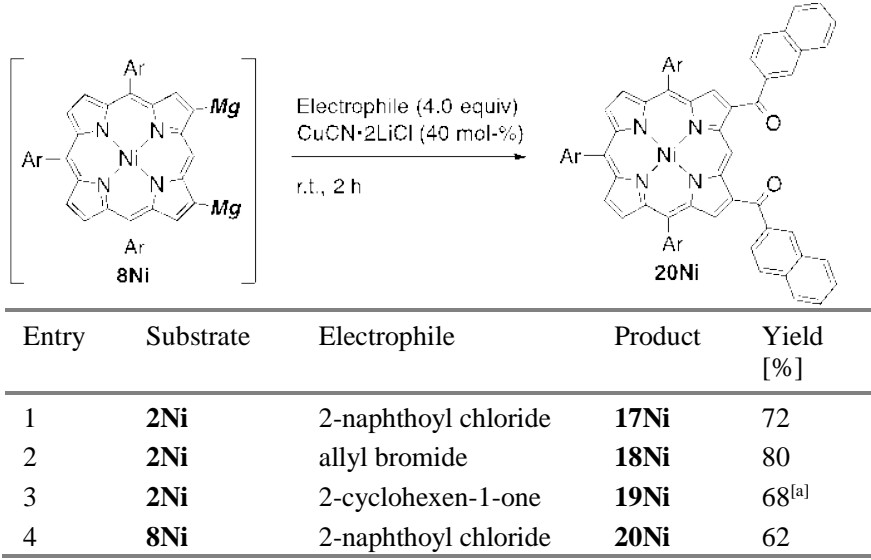

[a] With $\mathrm{Me}_{3} \mathrm{SiCl}$ (2 equiv).

Scheme 2. Negishi cross-coupling reactions of $\mathbf{2 1 N i}$

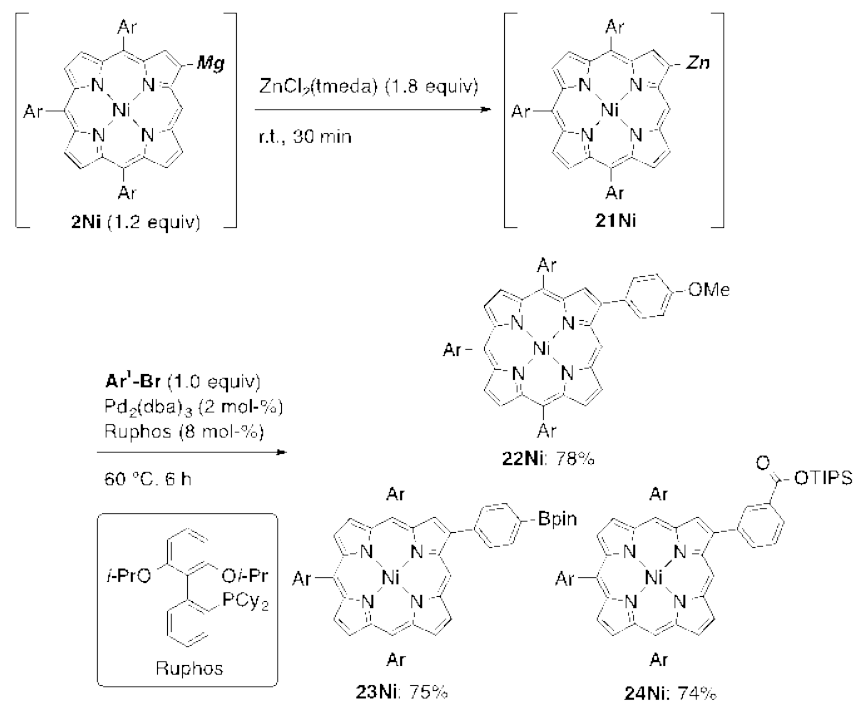

We finally examined Negishi cross-coupling reaction of porphyrinyl zinc species (Scheme 2). Porphyrinyl zinc 21Ni was prepared through transmetalation of porphyrinyl Grignard reagent 2Ni with $\mathrm{ZnCl}_{2}$ (tmeda) (tmeda = $N, N, N^{\prime}, N^{\prime}$ tetramethylethylenediamine). In the presence of $\mathrm{Pd}_{2}(\mathrm{dba})_{3} / 2-$ dicyclohexylphosphino-2',6'-diisopropoxybiphenyl (Ruphos) catalyst, ${ }^{[15]}$ Negishi cross-coupling reactions of $21 \mathrm{Ni}$ with 4- bromoanisole gave $\beta$-(4-anisyl)porphyrin $\mathbf{2 2 N i}$ in $78 \%$ yield. The high reactivity of organozinc reagents in transmetalation with an aryl palladium halide allows activator-free cross-coupling. With this advantage, 4-bromophenylboronate reacted chemoselectively to yield $23 \mathbf{N i}$ whereas the boronate moiety remained untouched. Furthermore, the low nucleophilicity of organozinc reagents toward carbonyl groups enabled the cross-coupling reaction of 21Ni with triisopropylsilyl 3-bromobenzoate without any observable nucleophilic attack.

\section{Conclusions}

We have successfully achieved the efficient synthesis of peripherally magnesiated porphyrins through iodine-magnesium exchange between iodoporphyrins with $\mathrm{iPrMgCl} \bullet \mathrm{LiCl}$ under mild conditions. The porphyrinyl Grignard reagents reacted with various carbonyl compounds as powerfully as the typical aryl Grignard reagents. Furthermore, transmetalation of the porphyrinyl Grignard reagents with copper and zinc salts efficiently proceeded. The resulting porphyrinyl copper and zinc were employed for their specific reactions, such as 1,4 -addition to enone and Negishi cross-coupling reaction, respectively. Further applications of the Grignard reagents to synthesize novel porphyrinoids are underway in our laboratory.

\section{Experimental Section}

Preparation of $\boldsymbol{i P r M g C l} \cdot \mathbf{L i C l}(1.0 \mathrm{M} \text { in THF })^{[11 a]}$ : A flask containing magnesium turnings $(0.67 \mathrm{~g}, 27.5 \mathrm{mmol})$ and anhydrous $\mathrm{LiCl}(1.06 \mathrm{~g}, 25$ mmol) was dried in vacuum ( $1-3$ torr) for $3 \mathrm{~h}$ at $150{ }^{\circ} \mathrm{C}$, and then purged with argon. After the flask was cooled to room temperature, dry THF (12 $\mathrm{mL})$ and 1,2-dibromoethane $(0.05 \mathrm{~mL})$ were added. A solution of $i \mathrm{PrCl}$ ( $2.28 \mathrm{~mL}, 25 \mathrm{mmol})$ in dry THF $(12 \mathrm{~mL}$ ) was then slowly added at room temperature. The reaction started within a few minutes. After the completion of the addition, the reaction mixture was stirred further for $12 \mathrm{~h}$ at room temperature. The resulting gray solution of $i \mathrm{PrMgCl} \cdot \mathrm{LiCl}$ was cannulated into another argon-filled Schlenk tube, being free from the remaining magnesium metal. The solution was stored at $-20^{\circ} \mathrm{C}$ and kept for at least 1 month without significant decomposition.

Synthesis of 3Ni-6Ni: A Schlenk tube containing $\mathrm{Ni}^{\mathrm{II}} \beta$-iodoporphyrin $\mathbf{1 N i}$ (106 mg, $100 \mu \mathrm{mol}$ ) was purged with argon, and then charged with dry THF $(2.0 \mathrm{~mL})$. After the solution was cooled to $-40{ }^{\circ} \mathrm{C}, i \mathrm{PrMgCl} \cdot \mathrm{LiCl}(1.0$ M solution in THF, $0.15 \mathrm{~mL}, 150 \mu \mathrm{mol}$ ) was slowly added, and then the reaction mixture was stirred for $2 \mathrm{~h}$ at $-40{ }^{\circ} \mathrm{C}$. To the resulting red solution, an electrophile (200 $\mu \mathrm{mol})$ was added. After being stirred for $2 \mathrm{~h}$ at room temperature, the reaction mixture was quenched with a sufficient amount of $\mathrm{NH}_{4} \mathrm{Cl}$ solution, extracted with $\mathrm{CH}_{2} \mathrm{Cl}_{2}$, washed with brine, and dried over $\mathrm{Na}_{2} \mathrm{SO}_{4}$. After removal of the solvent in vacuo, the residue was separated by silica gel chromatography eluting with $\mathrm{CH}_{2} \mathrm{Cl}_{2} /$ hexane. Recrystallization from $\mathrm{CH}_{2} \mathrm{Cl}_{2} /$ methanol gave $\mathbf{4 N i - 6 N i}$. For the synthesis of $\mathbf{3 N i}, \mathrm{D}_{2} \mathrm{O}$ (ca. $0.05 \mathrm{~mL}$ ) was added as an electrophile and the resulting mixture was stirred for $5 \mathrm{~min}$

3Ni: ${ }^{1} \mathrm{H}$ NMR (600 MHz, $\mathrm{CDCl}_{3}, 25^{\circ} \mathrm{C}$ ): $\delta=9.83$ (s, $1 \mathrm{H}$, meso), 9.13 (d, $J$ $=4.6 \mathrm{~Hz}, 1 \mathrm{H}, \beta), 8.93(\mathrm{~m}, 2 \mathrm{H}, \beta), 8.83(\mathrm{~m}, 4 \mathrm{H}, \beta), 7.90(\mathrm{~d}, J=1.8 \mathrm{~Hz}, 4 \mathrm{H}$, Ar-o), 7.87 (d, $J=1.8 \mathrm{~Hz}, 2 \mathrm{H}, \mathrm{Ar}-\mathrm{o}$ ), 7.74 (t, $J=1.8 \mathrm{~Hz}, 2 \mathrm{H}, \mathrm{Ar}-p), 7.71$ (t, $J=1.8 \mathrm{~Hz}, 1 \mathrm{H}, \mathrm{Ar}-p), 1.49$ (s, 36H, tert-butyl), and 1.46 (s, $18 \mathrm{H}$, tert-butyl) ppm; APCI-TOF-MS: $\mathrm{m} / \mathrm{z}=931.5174$. Calcd for $\mathrm{C}_{62} \mathrm{H}_{71} \mathrm{DN}_{4}{ }^{58} \mathrm{Ni}$ : 931.5168 $[\mathrm{M}]^{-}$.

4Ni: ${ }^{1} \mathrm{H}$ NMR (600 MHz, $\mathrm{CDCl}_{3}, 60^{\circ} \mathrm{C}$ ): $\delta=9.91$ (s, $1 \mathrm{H}$, meso), 9.04 (d, $J$ $=5.0 \mathrm{~Hz}, 1 \mathrm{H}, \beta), 8.86(\mathrm{~d}, J=5.0 \mathrm{~Hz}, 1 \mathrm{H}, \beta), 8.78(\mathrm{~s}, 4 \mathrm{H}, \beta), 8.75(\mathrm{~s}, 1 \mathrm{H}, \beta)$, 7.88 (d, $J=1.9 \mathrm{~Hz}, 2 \mathrm{H}$, Ar-o), 7.87 (br-s, 2H, Ar-o), 7.85 (d, $J=1.8 \mathrm{~Hz}$, 2H, Ar-o), 7.78 (d, $J=7.8 \mathrm{~Hz}, 2 \mathrm{H}, \mathrm{Ph}$ ), 7.75 (t, $J=1.9 \mathrm{~Hz}, 1 \mathrm{H}, \mathrm{Ar}-p), 7.73$ 
(t, $J=1.8 \mathrm{~Hz}, 1 \mathrm{H}, \mathrm{Ar}-p), 7.71(\mathrm{t}, J=1.8 \mathrm{~Hz}, 1 \mathrm{H}, \mathrm{Ar}-p), 7.40$ (m, 3H, $\mathrm{Ph}$ and benzyl), 7.32 (t, $J=7.8 \mathrm{~Hz}, 1 \mathrm{H}, \mathrm{Ph}$ ), 2.80 (d, $J=4.1 \mathrm{~Hz}, 1 \mathrm{H}, \mathrm{OH}), 1.49$ (s, 18H, tert-butyl) and 1.47 (s, 36H, tert-butyl) ppm; ${ }^{13} \mathrm{C}$ NMR $(151 \mathrm{MHz}$, $\left.\mathrm{CDCl}_{3}, 25^{\circ} \mathrm{C}\right): \delta=149.11,149.03,146.49,143.80,143.23,143.19,143.05$, 142.69, 141.16, 140.39, 140.20, 140.06, 139.96, 132.88, 132.51, 132.45, 132.33, 132.29, 131.07, 129.22, 128.92, 128.83, 128.05, 127.32, 121.24, 121.23, 120.89, 120.12, 120.09, 102.36, 72.04, 35.16, 35.14, 31.85, and 31.83 ppm; APCI-TOF-MS: $m / z=1036.5531$. Calcd for $\mathrm{C}_{69} \mathrm{H}_{78} \mathrm{ON}_{4}{ }^{58} \mathrm{Ni}$ : $1036.5524[\mathrm{M}]-; \mathrm{UV} / \mathrm{Vis}\left(\mathrm{CH}_{2} \mathrm{Cl}_{2}\right): \lambda_{\max }\left(\varepsilon\left[\mathrm{M}^{-1} \mathrm{Cm}^{-1}\right]\right)=413\left(2.6 \times 10^{5}\right)$ and $525\left(1.9 \times 10^{4}\right)$.

5Ni: ${ }^{1} \mathrm{H}$ NMR $\left(600 \mathrm{MHz}, \mathrm{CDCl}_{3}, 60^{\circ} \mathrm{C}\right): \delta=10.58$ (s, $1 \mathrm{H}$, meso), $9.14(\mathrm{~d}$, $J=4.6 \mathrm{~Hz}, 1 \mathrm{H}, \beta$ ), 8.88 (d, $J=4.6 \mathrm{~Hz}, 1 \mathrm{H}, \beta$ ), 8.77 (m, 5H, $\beta$ ), 7.90 (m, $4 \mathrm{H}$ Ar-o), 7.87 (d, $J=1.8 \mathrm{~Hz}, 2 \mathrm{H}, \mathrm{Ar}-\mathrm{o}$ ), 7.75 (m, 2H, Ar-p), 7.72 (t, $J=1.8$ $\mathrm{Hz}, 1 \mathrm{H}, \mathrm{Ar}-p), 2.72$ (d, $J=13.3 \mathrm{~Hz}, 2 \mathrm{H}$, cyclohexyl), 2.55-2.49 (m, 2H, cyclohexyl), 2.49 (s, 1H, OH), 2.19-2.13 (m, 2H, cyclohexyl), 1.93-1.85 (m, 3H, cyclohexyl), 1.59-1.52 (m, 1H, cyclohexyl), 1.51 (s, 18H, tertbutyl), 1.50 (s, $18 \mathrm{H}$, tert-butyl), and 1.47 (s, $18 \mathrm{H}$, tert-butyl) ppm; ${ }^{13} \mathrm{C}$ NMR (151 MHz, $\left.\mathrm{CDCl}_{3}, 25^{\circ} \mathrm{C}\right): \delta=151.76,149.13,149.10,149.02$, 143.03, 142.93, 142.88, 142.54, 142.49, 142.43, 140.64, 140.52, 140.28, $140.23,140.12,132.74,132.55,132.43,132.26,132.21,129.28,128.92$, $128.83,121.24,121.18,121.11,120.49,119.92,119.40,104.83,73.12$, 40.80, 35.19, 35.16, 35.13, 31.87, 31.84, 26.11, and 22.76 ppm; APCITOF-MS: $\mathrm{m} / \mathrm{z}=1028.5846$. Calcd for $\mathrm{C}_{68} \mathrm{H}_{82} \mathrm{ON}_{4}{ }^{58} \mathrm{Ni}: 1028.5837[\mathrm{M}]^{-}$; UV/Vis $\left(\mathrm{CH}_{2} \mathrm{Cl}_{2}\right): \lambda_{\max }\left(\varepsilon\left[\mathrm{M}^{-1} \mathrm{~cm}^{-1}\right]\right)=413\left(2.6 \times 10^{5}\right)$ and $524\left(1.9 \times 10^{4}\right)$. 6Ni: ${ }^{1} \mathrm{H}$ NMR (600 MHz, $\mathrm{CDCl}_{3}, 25^{\circ} \mathrm{C}$ ): $\delta=11.01$ (s, $1 \mathrm{H}$, formyl), 10.69 (s, $1 \mathrm{H}$, meso), 9.37 (s, $1 \mathrm{H}, \beta$ ), 9.17 (d, $J=4.6 \mathrm{~Hz}, 1 \mathrm{H}, \beta$ ), 8.86 (d, $J=4.6$ $\mathrm{Hz}, 1 \mathrm{H}, \beta$ ), 8.78 (m, 3H, $\beta$ ), 8.74 (d, $J=4.9 \mathrm{~Hz}, 1 \mathrm{H}, \beta$ ), 7.88 (s, $2 \mathrm{H}, \mathrm{Ar}-\mathrm{o}$ ), 7.86 (s, 2H, Ar-o), 7.83 (s, 2H, Ar-o), 7.78 (s, 1H, Ar-p), 7.74 (s, 1H, Ar-p), 7.72 (s, $1 \mathrm{H}$, Ar-p), 1.50 (s, 18H, tert-butyl), 1.49 (s, 18H, tert-butyl), and 1.46 (s, 18H, tert-butyl) ppm; ${ }^{13} \mathrm{C}$ NMR $\left(151 \mathrm{MHz}, \mathrm{CDCl}_{3}, 25{ }^{\circ} \mathrm{C}\right): \delta$ $=188.04,149.31,149.21,144.72,144.64,144.41,143.75,143.50,142.76$, $140.20,139.77,139.63,139.52,139.47,139.34,137.32,134.00,133.50$, 133.46, 133.21, 133.06, 132.54, 128.87, 128.72, 122.99, 121.79, 121.43, 121.22, 119.84, 104.71, 35.18, 35.17, 35.14, 31.83, and 31.80 ppm; APCITOF-MS: $\mathrm{m} / \mathrm{z}=958.5080$. Calcd for $\mathrm{C}_{63} \mathrm{H}_{72} \mathrm{ON}_{4}{ }^{58} \mathrm{Ni}$ : $958.5054[\mathrm{M}]^{-}$; UV/Vis $\left(\mathrm{CH}_{2} \mathrm{Cl}_{2}\right): \lambda_{\max }\left(\varepsilon\left[\mathrm{M}^{-1} \mathrm{~cm}^{-1}\right]\right)=426\left(2.1 \times 10^{5}\right), 535\left(1.3 \times 10^{4}\right)$, and $577\left(1.2 \times 10^{4}\right)$.

Synthesis of $\mathbf{3 Z n}$ and $\mathbf{5 Z n}$ : This procedure is similar to that for the synthesis of $\mathbf{3 N i - 6 N i ~ e x c e p t ~ t h a t ~ i o d i n e - m a g n e s i u m ~ e x c h a n g e ~ o f ~} \mathbf{1 Z n}$ was performed at $-80{ }^{\circ} \mathrm{C}$. Recrystallization from $\mathrm{CH}_{2} \mathrm{Cl}_{2} /$ methanol gave $3 \mathbf{Z n}$ (85 mg, $90 \mu \mathrm{mol}, 90 \%$ ) and 5Zn (70 mg, $68 \mu \mathrm{mol}, 68 \%$ ).

3Zn: ${ }^{1} \mathrm{H}$ NMR (600 MHz, $\mathrm{CDCl}_{3}, 25^{\circ} \mathrm{C}$ ): $\delta=10.27$ (s, $1 \mathrm{H}$, meso), 9.42 (d, $J=4.7 \mathrm{~Hz}, 1 \mathrm{H}, \beta$ ), 8.93 (m, 2H, $\beta$ ), 9.06 (d, $J=4.6 \mathrm{~Hz}, 2 \mathrm{H}, \beta$ ), 9.03 (d, $J=$ $4.6 \mathrm{~Hz}, 2 \mathrm{H}, \beta$ ), 8.12 (d, $J=1.9 \mathrm{~Hz}, 4 \mathrm{H}, \mathrm{Ar}-\mathrm{o}$ ), 8.09 (d, $J=2.0 \mathrm{~Hz}, 2 \mathrm{H}, \mathrm{Ar}-$ o), 7.82 (t, $J=1.9 \mathrm{~Hz}, 2 \mathrm{H}, \mathrm{Ar}-p), 7.79$ (t, $J=2.0 \mathrm{~Hz}, 1 \mathrm{H}, \mathrm{Ar}-p), 1.55$ (s, $36 \mathrm{H}$, tert-butyl) and 1.52 (s, $18 \mathrm{H}$, tert-butyl) ppm; APCI-TOF-MS: $\mathrm{m} / \mathrm{z}=$ 937.5120. Calcd for $\mathrm{C}_{62} \mathrm{H}_{71} \mathrm{DN}_{4}{ }^{64} \mathrm{Zn}$ : $937.5106[\mathrm{M}]^{-}$.

5Zn: ${ }^{1} \mathrm{H}$ NMR (600 MHz, $\left.\mathrm{CDCl}_{3}, 60{ }^{\circ} \mathrm{C}\right): \delta=10.84$ (s, $1 \mathrm{H}$, meso), $9.41(\mathrm{~d}$, $J=4.6 \mathrm{~Hz}, 1 \mathrm{H}, \beta), 9.11$ (d, $J=4.6 \mathrm{~Hz}, 1 \mathrm{H}, \beta), 9.03$ (m, 3H, $\beta$ ), 8.99 (d, $J=$ $4.6 \mathrm{~Hz}, 1 \mathrm{H}, \beta$ ), 8.85 (s, $1 \mathrm{H}, \beta), 8.12$ (m, 4H, Ar-o), 8.09 (d, $J=1.8 \mathrm{~Hz}, 2 \mathrm{H}$, Ar-o), 7.83 (m, 2H, Ar-p), 7.81 (br-s, 1H, Ar-p), 2.72 (d, $J=13.3 \mathrm{~Hz}, 2 \mathrm{H}$, cyclohexyl), 2.55-2.49 (m, 2H, cyclohexyl), 2.43 (s, 1H, OH), 2.14-2.09 (m, 2H, cyclohexyl), 1.94-1.86 (m, 3H, cyclohexyl), 1.58 (s, 18H, tertbutyl), 1.56 (s, $18 \mathrm{H}$, tert-butyl), and 1.54 (s, $18 \mathrm{H}$, tert-butyl) ppm; ${ }^{13} \mathrm{C}$ NMR (151 MHz, $\left.\mathrm{CDCl}_{3}, 60{ }^{\circ} \mathrm{C}\right): \delta=150.76,150.68,150.30,150.14$, $149.71,148.91,148.88,148.78,148.31,147.78,142.39,142.22,142.13$, $132.60,132.40,132.27,132.20,132.08,131.93,130.37,129.85,129.74$, 129.27, 122.84, 122.17, 121.67, 121.02, 120.99, 120.81, 106.14, 73.24, 41.07, 35.31, 35.28, 35.21, 32.00, 21.97, 26.13, and 22.79 ppm; APCITOF-MS: $m / z=1034.5788$. Calcd for $\mathrm{C}_{68} \mathrm{H}_{82} \mathrm{ON}_{4}{ }^{64} \mathrm{Zn}$ : $1034.5775[\mathrm{M}]^{-}$; $\mathrm{UV} / \mathrm{Vis}\left(\mathrm{CH}_{2} \mathrm{Cl}_{2}\right): \lambda_{\max }\left(\varepsilon\left[\mathrm{M}^{-1} \mathrm{Cm}^{-1}\right]\right)=418\left(6.1 \times 10^{5}\right)$ and $545\left(2.4 \times 10^{4}\right)$.
Synthesis of 9Ni and 10Ni: A Schlenk tube containing $\mathrm{Ni}^{\mathrm{II}} \beta, \beta$ 'diiodoporphyrin $7 \mathrm{Ni}(118 \mathrm{mg}, 100 \mu \mathrm{mol})$ was purged with argon, and then charged with dry THF $(2.0 \mathrm{~mL})$. After the solution was cooled to $-40{ }^{\circ} \mathrm{C}$, $i \mathrm{PrMgCl} \cdot \mathrm{LiCl}(1.0 \mathrm{M}$ solution in THF, $0.30 \mathrm{~mL}, 300 \mu \mathrm{mol})$ was slowly added, and then the reaction mixture was stirred for $2 \mathrm{~h}$ at $-40{ }^{\circ} \mathrm{C}$. To the resulting red solution, DMF ( $32 \mu \mathrm{L}, 400 \mu \mathrm{mol}$ ) was added. After being stirred for $2 \mathrm{~h}$ at room temperature, the reaction mixture was quenched with a sufficient amount of $\mathrm{NH}_{4} \mathrm{Cl}$ solution, extracted with $\mathrm{CH}_{2} \mathrm{Cl}_{2}$, washed with brine, and dried over $\mathrm{Na}_{2} \mathrm{SO}_{4}$. After removal of the solvent in vacuo, the residue was separated by silica gel chromatography eluting with $\mathrm{CH}_{2} \mathrm{Cl}_{2}$ /hexane. Recrystallization from $\mathrm{CH}_{2} \mathrm{Cl}_{2} /$ methanol gave 10Ni (76 mg, $77 \mu \mathrm{mol}, 77 \%$ ). For the synthesis of $\mathbf{9 N i}$ (88 mg, $94 \mu \mathrm{mol}, 94 \%$ ), $\mathrm{D}_{2} \mathrm{O}$ (ca. $0.1 \mathrm{~mL}$ ) was added instead of DMF and the resulting mixture was stirred for $5 \mathrm{~min}$.

9Ni: ${ }^{1} \mathrm{H}$ NMR (600 MHz, $\mathrm{CDCl}_{3}, 25^{\circ} \mathrm{C}$ ): $\delta=9.83$ (s, $1 \mathrm{H}$, meso), 8.93 (s, $2 \mathrm{H}, \beta$ ), 8.83 (m, 4H, $\beta$ ), 7.90 (d, $J=1.8 \mathrm{~Hz}, 4 \mathrm{H}, \mathrm{Ar}-o$ ), 7.87 (d, $J=1.8 \mathrm{~Hz}$, $2 \mathrm{H}, \mathrm{Ar}-o$ ), 7.74 (t, $J=1.8 \mathrm{~Hz}, 2 \mathrm{H}, \mathrm{Ar}-p$ ), 7.71 (t, $J=1.8 \mathrm{~Hz}, 1 \mathrm{H}, \mathrm{Ar}-p$ ), 1.49 (s, 36H, tert-butyl) and 1.46 (s, 18H, tert-butyl) ppm; APCI-TOF-MS: $m / z=932.5235$. Calcd for $\mathrm{C}_{62} \mathrm{H}_{70} \mathrm{D}_{2} \mathrm{~N}_{4}{ }^{58} \mathrm{Ni}: 932.5231[\mathrm{M}]^{-}$.

10Ni: ${ }^{1} \mathrm{H}$ NMR (600 MHz, $\mathrm{CDCl}_{3}, 25^{\circ} \mathrm{C}$ ): $\delta=11.38$ (s, $1 \mathrm{H}$, meso), 11.16 (s, $1 \mathrm{H}$, formyl), 9.38 (s, $2 \mathrm{H}, \beta$ ), 8.77 (d, $J=4.6 \mathrm{~Hz}, 2 \mathrm{H}, \beta$ ), 8.75 (d, $J=4.6 \mathrm{~Hz}$, $2 \mathrm{H}, \beta$ ), 7.84 (d, $J=1.8 \mathrm{~Hz}, 4 \mathrm{H}, \mathrm{Ar}-\mathrm{o}$ ), 7.81 (d, $J=1.8 \mathrm{~Hz}, 2 \mathrm{H}, \mathrm{Ar}-o$ ), 7.73 (t, J = 1.8 Hz, 2H, Ar-p), 7.73 (t, J = 1.8 Hz, 1H, Ar-p), 1.50 (s, 36H, tertbutyl), and 1.46 (s, $18 \mathrm{H}$, tert-butyl) ppm; ${ }^{13} \mathrm{C}$ NMR (151 MHz, $\mathrm{CDCl}_{3}$, $\left.25^{\circ} \mathrm{C}\right): \delta=187.45,149.54,149.41,144.83,144.15,140.90,140.10,139.59$, 139.32, 139.00, 138.76, 133.81, 133.59, 128.84, 128.64, 122.85, 122.02, 121.69, 103.76, 35.19, 35.16, 31.82 and 31.80 ppm; APCI-TOF-MS: $\mathrm{m} / \mathrm{z}=$ 986.5032. Calcd for $\mathrm{C}_{64} \mathrm{H}_{72} \mathrm{O}_{2} \mathrm{~N}_{4}{ }^{58} \mathrm{Ni}$ : $986.5003[\mathrm{M}]$; UV/Vis $\left(\mathrm{CH}_{2} \mathrm{Cl}_{2}\right)$ : $\lambda_{\max }\left(\varepsilon\left[\mathrm{M}^{-1} \mathrm{Cm}^{-1}\right]\right)=441\left(2.0 \times 10^{5}\right), 551\left(1.4 \times 10^{4}\right)$, and $592\left(1.1 \times 10^{4}\right)$.

Synthesis of 13Ni-16Ni: This procedure is similar to that for the synthesis of $\mathbf{3 N i - 6 N i ~ e x c e p t ~ f o r ~ t h e ~ s t a r t i n g ~ m a t e r i a l . ~}$

13Ni: ${ }^{1} \mathrm{H}$ NMR ( $600 \mathrm{MHz}, \mathrm{CDCl}_{3}, 25^{\circ} \mathrm{C}$ ): $\delta=9.14(\mathrm{~d}, J=4.6 \mathrm{~Hz}, 2 \mathrm{H}, \beta)$, 8.93 (d, $J=4.6 \mathrm{~Hz}, 2 \mathrm{H}, \beta$ ), 8.84 (m, 4H, $\beta$ ), 7.90 (d, $J=1.8 \mathrm{~Hz}, 4 \mathrm{H}, \mathrm{Ar}-\mathrm{o}$ ), 7.88 (d, $J=1.8 \mathrm{~Hz}, 2 \mathrm{H}, \mathrm{Ar}-o$ ), 7.74 (t, $J=1.8 \mathrm{~Hz}, 2 \mathrm{H}, \mathrm{Ar}-p), 7.71$ (t, $J=$ $1.8 \mathrm{~Hz}, 1 \mathrm{H}$, Ar-p), 1.49 (s, 36H, tert-butyl) and 1.46 (s, 18H, tert-butyl) ppm; APCI-TOF-MS: $m / z=931.5196$. Calcd for $\mathrm{C}_{62} \mathrm{H}_{71} \mathrm{DN}_{4}{ }^{58} \mathrm{Ni}$ : 931.5168 $[\mathrm{M}]^{-}$.

14Ni: ${ }^{1} \mathrm{H}$ NMR ( $\left.600 \mathrm{MHz}, \mathrm{CDCl}_{3}, 25{ }^{\circ} \mathrm{C}\right): \delta=9.28(\mathrm{~d}, J=5.0 \mathrm{~Hz}, 2 \mathrm{H}, \beta)$, $8.76(\mathrm{~m}, 4 \mathrm{H}, \beta), 8.74(\mathrm{~d}, J=4.6 \mathrm{~Hz}, 2 \mathrm{H}, \beta), 8.01$ (d, $J=3.7 \mathrm{~Hz}, 1 \mathrm{H}$, benzyl), 7.83 (d, $J=1.8 \mathrm{~Hz}, 2 \mathrm{H}$, Ar-o), 7.81 (d, $J=1.3 \mathrm{~Hz}, 4 \mathrm{H}$, Ar-o), 7.69 (s, 3H, Ar-p), 7.57 (d, $J=7.8 \mathrm{~Hz}, 2 \mathrm{H}, \mathrm{Ph}$ ), 7.28 (d, $J=7.8 \mathrm{~Hz}, 2 \mathrm{H}, \mathrm{Ph}$ ), 7.23 (d, $J=7.8 \mathrm{~Hz}, 1 \mathrm{H}, \mathrm{Ph}$ ), 3.36 (d, $J=3.7 \mathrm{~Hz}, 1 \mathrm{H}, \mathrm{OH}$ ), and 1.45 (s, $54 \mathrm{H}$, tert-butyl) ppm; ${ }^{13} \mathrm{C}$ NMR (151 MHz, $\left.\mathrm{CDCl}_{3}, 25{ }^{\circ} \mathrm{C}\right): \delta=149.12,147.02$, $142.81,142.40,142.02,141.82,139.3,133.63,132.83,132.44,130.62$, 128.70, 128.26, 126.82, 126.51, 121.31, 120.85, 120.04, 116.58, 75.13, 35.12, and $31.80 \mathrm{ppm}$; APCI-TOF-MS: $\mathrm{m} / \mathrm{z}=1036.5546$. Calcd for $\mathrm{C}_{64} \mathrm{H}_{72} \mathrm{O}_{2} \mathrm{~N}_{4}{ }^{58} \mathrm{Ni}: 1036.5524[\mathrm{M}]^{-}$; UV/Vis $\left(\mathrm{CH}_{2} \mathrm{Cl}_{2}\right): \lambda_{\max }\left(\varepsilon\left[\mathrm{M}^{-1} \mathrm{Cm}^{-1}\right]\right)=$ $418\left(2.5 \times 10^{5}\right)$ and $533\left(1.7 \times 10^{4}\right)$.

15Ni: ${ }^{1} \mathrm{H}$ NMR (600 MHz, $\left.\mathrm{CDCl}_{3}, 25{ }^{\circ} \mathrm{C}\right): \delta=9.67(\mathrm{~d}, J=5.0 \mathrm{~Hz}, 2 \mathrm{H}, \beta)$, 8.67 (m, 4H, $\beta$ ), 8.60 (d, $J=5.0 \mathrm{~Hz}, 2 \mathrm{H}, \beta$ ), 7.79 (br-s, 2H, Ar-o), 7.76 (brs, $4 \mathrm{H}, \mathrm{Ar}-\mathrm{o}$ ), 7.67 (m, 3H, Ar-p), 3.37 (m, 2H, cyclohexyl), 2.46 (d, $J=$ $14.2 \mathrm{~Hz}, 2 \mathrm{H}$, cyclohexyl), 2.14-2.06 (m, 2H, cyclohexyl), 2.01 (br-d, 1H, cyclohexyl), 1.91 (m, 2H, cyclohexyl), 1.76 (m, 1H, cyclohexyl), 1.58 (s, 1H, OH), 1.45 (s, 36H, tert-butyl), and 1.43 (s, 18H, tert-butyl) ppm; ${ }^{13} \mathrm{C}$ NMR (151 MHz, $\left.\mathrm{CDCl}_{3}, 25{ }^{\circ} \mathrm{C}\right): \delta=149.10,141.99,141.45,139.90$, 139.61, 139.47, 139.42, 133.66, 132.68, 132.63, 132.04, 128.62, 122.53, $121.18,120.34,119.20,44.86,35.10,31.79,25.77$, and 23.18 ppm; APCITOF-MS: $m / z=1028.5865$. Calcd for $\mathrm{C}_{68} \mathrm{H}_{82} \mathrm{ON}_{4}{ }^{58} \mathrm{Ni}$ : $1028.5837[\mathrm{M}]$; UV/Vis $\left(\mathrm{CH}_{2} \mathrm{Cl}_{2}\right): \lambda_{\max }\left(\varepsilon\left[\mathrm{M}^{-1} \mathrm{Cm}^{-1}\right]\right)=419\left(2.4 \times 10^{5}\right)$ and $533\left(1.6 \times 10^{4}\right)$. 16Ni: ${ }^{1} \mathrm{H}$ NMR (600 MHz, $\mathrm{CDCl}_{3}, 25^{\circ} \mathrm{C}$ ): $\delta=12.05$ (s, $1 \mathrm{H}$, formyl), 9.79 (d, $J=5.3 \mathrm{~Hz}, 2 \mathrm{H}, \beta$ ), 8.88 (d, $J=5.3 \mathrm{~Hz}, 2 \mathrm{H}, \beta$ ), 8.69 (d, $J=4.7 \mathrm{~Hz}, 2 \mathrm{H}$, $\beta$ ), 8.62 (d, $J=4.6 \mathrm{~Hz}, 2 \mathrm{H}, \beta$ ), 7.80 (m, $6 \mathrm{H}, \mathrm{Ar}-\mathrm{o}$ ), 7.73 (t, $J=1.8 \mathrm{~Hz}, 2 \mathrm{H}$, 
Ar-p), 7.73 (t, $J=1.9 \mathrm{~Hz}, 1 \mathrm{H}, \mathrm{Ar}-p$ ), 1.47 (s, 36H, tert-butyl), and 1.45 (s, $18 \mathrm{H}$, tert-butyl) ppm; ${ }^{13} \mathrm{C}$ NMR (151 MHz, $\left.\mathrm{CDCl}_{3}, 25{ }^{\circ} \mathrm{C}\right): \delta=192.89$, $149.39,149.31,144.75,144.54,142.08,141.16,139.16,135.83,133.69$, 132.25, 130.63, 128.61, 128.49, 124.94, 122.39, 121.66, 105.87, 35.15, 31.80, and $31.78 \mathrm{ppm}$; APCI-TOF-MS: $\mathrm{m} / \mathrm{z}=958.5072$. Calcd for $\mathrm{C}_{63} \mathrm{H}_{72} \mathrm{ON}_{4}{ }^{58} \mathrm{Ni}$ : $958.5054[\mathrm{M}]^{-}$; UV/Vis $\left(\mathrm{CH}_{2} \mathrm{Cl}_{2}\right): \lambda_{\max }\left(\varepsilon\left[\mathrm{M}^{-1} \mathrm{~cm}^{-1}\right]\right)=427$ $\left(2.1 \times 10^{5}\right)$, $554\left(1.0 \times 10^{4}\right)$, and $596\left(1.5 \times 10^{4}\right)$.

Synthesis of 13Zn, 15Zn and 16Zn: This procedure is similar to that for the synthesis of $\mathbf{3 Z n}$ and $\mathbf{5 Z n}$ except for the starting material.

13Zn: ${ }^{1} \mathrm{H}$ NMR (600 MHz, $\left.\mathrm{CDCl}_{3}, 25^{\circ} \mathrm{C}\right): \delta=9.42(\mathrm{~d}, J=4.7 \mathrm{~Hz}, 1 \mathrm{H}, \beta)$, 9.15 (d, $J=4.7 \mathrm{~Hz}, 2 \mathrm{H}, \beta), 9.06$ (d, $J=4.6 \mathrm{~Hz}, 2 \mathrm{H}, \beta$ ), 9.03 (d, $J=4.6 \mathrm{~Hz}$, $2 \mathrm{H}, \beta$ ), 8.12 (d, $J=1.9 \mathrm{~Hz}, 4 \mathrm{H}, \mathrm{Ar}-\mathrm{o}$ ), 8.09 (d, $J=2.0 \mathrm{~Hz}, 2 \mathrm{H}, \mathrm{Ar}-\mathrm{o}$ ), 7.82 (t, $J=1.9 \mathrm{~Hz}, 2 \mathrm{H}, \mathrm{Ar}-p), 7.79$ (t, $J=2.0 \mathrm{~Hz}, 1 \mathrm{H}, \mathrm{Ar}-p), 1.55$ (s, 36H, tertbutyl) and 1.52 (s, 18H, tert-butyl) ppm; APCI-TOF-MS: $m / z=937.5133$. Calcd for $\mathrm{C}_{62} \mathrm{H}_{71} \mathrm{DN}_{4}{ }^{64} \mathrm{Zn}$ : $937.5106[\mathrm{M}]^{-}$.

15Zn: ${ }^{1} \mathrm{H}$ NMR (600 MHz, $\left.\mathrm{CDCl}_{3}, 25^{\circ} \mathrm{C}\right): \delta=10.08(\mathrm{~d}, J=4.6 \mathrm{~Hz}, 2 \mathrm{H}, \beta)$, 8.90 (d, $J=4.6 \mathrm{~Hz}, 2 \mathrm{H}, \beta), 8.84$ (m, 4H, $\beta$ ), 8.04 (d, $J=1.9 \mathrm{~Hz}, 2 \mathrm{H}, \mathrm{Ar}-\mathrm{o}$ ), 8.03 (d, $J=1.9 \mathrm{~Hz}, 4 \mathrm{H}, \mathrm{Ar}-\mathrm{o}$ ), 7.78 (br-s, 2H, Ar-p), 7.75 (br-s, 1H, Ar-p), 3.83 (m, 2H, cyclohexyl), 2.76 (d, $J=14.7 \mathrm{~Hz}, 2 \mathrm{H}$, cyclohexyl), 2.34 (s, $1 \mathrm{H}$ $\mathrm{OH}), 2.30$ (m, 2H, cyclohexyl), 2.12 (m, 1H, cyclohexyl), 2.05 (m, $2 \mathrm{H}$, cyclohexyl), 1.96 (m, 1H, cyclohexyl), 1.53 (s, 36H, tert-butyl), and 1.51 (s, $18 \mathrm{H}$, tert-butyl) ppm; ${ }^{13} \mathrm{C}$ NMR $\left(151 \mathrm{MHz}, \mathrm{CDCl}_{3}, 25{ }^{\circ} \mathrm{C}\right): \delta=150.33$, $150.00,149.07,148.90,148.86,148.65,142.23,141.89,132.17,131.76$, 130.93, 129.70, 129.63, 122.98, 122.22, 121.00, 79.35, 46.27, 35.25, 31.97, 31.94, 25.88, and 23.80 ppm; APCI-TOF-MS: $\mathrm{m} / \mathrm{z}=1034.5760$. Calcd for $\mathrm{C}_{68} \mathrm{H}_{82} \mathrm{ON}_{4}{ }^{64} \mathrm{Zn}: 1034.5775[\mathrm{M}]^{-}$; UV/Vis $\left(\mathrm{CH}_{2} \mathrm{Cl}_{2}\right): \lambda_{\max }\left(\varepsilon\left[\mathrm{M}^{-1} \mathrm{~cm}^{-1}\right]\right)=$ $424\left(4.0 \times 10^{5}\right)$ and $557\left(1.8 \times 10^{4}\right)$.

16Zn: ${ }^{1} \mathrm{H}$ NMR $\left(600 \mathrm{MHz}, \mathrm{CDCl}_{3}, 60{ }^{\circ} \mathrm{C}\right.$ ): $\delta=12.28-12.20$ (br-s, $1 \mathrm{H}$, formyl), 9.93 (br-s, $2 \mathrm{H}, \beta$ ), 9.07 (d, $J=5.0 \mathrm{~Hz}, 2 \mathrm{H}, \beta$ ), 8.90 (d, $J=4.6 \mathrm{~Hz}$, $2 \mathrm{H}, \beta$ ), 8.84 (d, $J=4.6 \mathrm{~Hz}, 2 \mathrm{H}, \beta$ ), 8.03 (d, $J=1.9 \mathrm{~Hz}, 4 \mathrm{H}, \mathrm{Ar}-0$ ), 8.01 (d, $J$ $=1.8 \mathrm{~Hz}, 2 \mathrm{H}, \mathrm{Ar}-o$ ), 7.81 (br-s, 2H, Ar-p), 7.78 (br-s, 1H, Ar-p), 1.53 (s, $36 \mathrm{H}$, tert-butyl), and 1.50 (s, $18 \mathrm{H}$, tert-butyl) ppm; ${ }^{13} \mathrm{C} \mathrm{NMR}(151 \mathrm{MHz}$, $\left.\mathrm{CDCl}_{3}, 60^{\circ} \mathrm{C}\right): \delta=195.23,153.35,152.29,149.66,149.26,148.88,148.74$, $141.45,141.32$, 135.08, 133.40, 131.77, 129.51, 129.44, 128.76, 128.53, 125.42, 121.29, 35.20, 35.14, 31.93, and 31.83 ppm; APCI-TOF-MS: $m / z=$ 964.5010. Calcd for $\mathrm{C}_{63} \mathrm{H}_{72} \mathrm{ON}_{4}{ }^{64} \mathrm{Zn}$ : $964.4992[\mathrm{M}]^{-}$; UV/Vis $\left(\mathrm{CH}_{2} \mathrm{Cl}_{2}\right): \lambda_{\max }$ $\left(\varepsilon\left[\mathrm{M}^{-1} \mathrm{~cm}^{-1}\right]\right)=429\left(4.3 \times 10^{5}\right), 560\left(1.5 \times 10^{4}\right)$, and $604\left(2.1 \times 10^{4}\right)$.

Preparation of $\mathrm{CuCN} \cdot 2 \mathrm{LiCl}(0.2 \mathrm{M} \text { in THF })^{[16]}$ : A Schlenk tube containing $\mathrm{CuCN}$ (36 mg, $0.40 \mathrm{mmol}$ ) and anhydrous $\mathrm{LiCl}$ (34 mg, 0.80 $\mathrm{mmol}$ ) was dried in vacuum (1-3 torr) for $3 \mathrm{~h}$ at $150^{\circ} \mathrm{C}$, and then purged with argon. After the flask was cooled to room temperature, THF $(2.0 \mathrm{~mL})$ was added. After the reaction mixture was stirred for $30 \mathrm{~min}$ at room temperature, a yellow solution of $\mathrm{CuCN} \cdot 2 \mathrm{LiCl}$ was obtained.

Synthesis of $\mathbf{1 7 N i}$ and 18Ni: After $\mathbf{2 N i}$ was generated as described in the synthesis of $\mathbf{3 N i - 6 N i , ~ C u C N} \cdot 2 \mathrm{LiCl}(0.2 \mathrm{M}$ solution in THF, $0.10 \mathrm{~mL}, 20$ $\mu \mathrm{mol})$ and an electrophile $(200 \mu \mathrm{mol})$ were sequentially added. After being stirred for $2 \mathrm{~h}$ at room temperature, the reaction mixture was quenched with an $\mathrm{NH}_{4} \mathrm{Cl}$ solution, extracted with $\mathrm{CH}_{2} \mathrm{Cl}_{2}$, washed with brine, and dried over $\mathrm{Na}_{2} \mathrm{SO}_{4}$. After evaporation, the residue was purified on silica gel eluting with $\mathrm{CH}_{2} \mathrm{Cl}_{2} /$ hexane. Recrystallization from $\mathrm{CH}_{2} \mathrm{Cl}_{2} /$ methanol gave 17Ni (78 mg, $72 \mu \mathrm{mol}, 72 \%$ ) and 18Ni (78 mg, $80 \mu \mathrm{mol}, 80 \%$ ).

17Ni: ${ }^{1} \mathrm{H}$ NMR (600 MHz, $\mathrm{CDCl}_{3}, 25^{\circ} \mathrm{C}$ ): $\delta=10.56$ (s, $1 \mathrm{H}$, meso), 9.16 (m, $2 \mathrm{H}, \beta$ ), 8.88 (d, $J=4.6 \mathrm{~Hz}, 1 \mathrm{H}, \beta$ ), 8.81 (m, 3H, $\beta$ ), 8.78 (d, $J=4.8 \mathrm{~Hz}, 1 \mathrm{H}$, $\beta$ ), 8.71 (s, $1 \mathrm{H}$, naphthyl), 8.38 (d, $J=8.2 \mathrm{~Hz}, 1 \mathrm{H}$, naphthyl), 8.06 (d, $J=$ $8.2 \mathrm{~Hz}, 1 \mathrm{H}$, naphthyl), 7.98 (d, $J=8.2 \mathrm{~Hz}, 1 \mathrm{H}$, naphthyl), 7.92 (d, $J=8.2$ $\mathrm{Hz}, 1 \mathrm{H}$, naphthyl), 7.90 (d, 2H, $J=1.9 \mathrm{~Hz}, \mathrm{Ar}-\mathrm{o}$ ), 7.90 (d, $J=1.8 \mathrm{~Hz}, 2 \mathrm{H}$, Ar-o), 7.90 (d, $J=1.8 \mathrm{~Hz}, 2 \mathrm{H}, \mathrm{Ar}-\mathrm{o}$ ), 7.74 (t, $J=1.9 \mathrm{~Hz}, 1 \mathrm{H}, \mathrm{Ar}-p$ ), 7.72 (t, $J=1.8 \mathrm{~Hz}, 1 \mathrm{H}, \mathrm{Ar}-p), 7.65$ (t, $J=8.7 \mathrm{~Hz}, 1 \mathrm{H}$, naphthyl), 7.63 (t, $J=1.8 \mathrm{~Hz}$, $1 \mathrm{H}, \mathrm{Ar}-p$ ), 7.56 (t, $J=8.7 \mathrm{~Hz}, 1 \mathrm{H}$, naphthyl), 1.49 (s, 18H, tert-butyl), 1.47 (s, 18H, tert-butyl), and 1.41 (s, 18H, tert-butyl) ppm; ${ }^{13} \mathrm{C}$ NMR (151 MHz,
$\left.\mathrm{CDCl}_{3}, 25^{\circ} \mathrm{C}\right): \delta=193.40,149.27,149.16,144.27,143.97,143.33,142.73$, 142.82, 141.05, 139.94, 139.76, 139.63, 139.18, 138.06, 137.61, 137.50, 135.60, 133.96, 133.25, 132.94, 132.65, 132.50, 132.41, 129.90, 128.93, $128.84,128.76,128.59,128.52,127.99,126.86,126.15,122.25,121.53$, 121.41, 120.97, 119.82, 105.19, 35.17, 35.15, 35.08, 31.84, 31.82, and 31.79 ppm; APCI-TOF-MS: $m / z=1084.5533$. Calcd for $\mathrm{C}_{73} \mathrm{H}_{78} \mathrm{ON}_{4}{ }^{58} \mathrm{Ni}$ : 1084.5524 [M]-; UV/Vis $\left(\mathrm{CH}_{2} \mathrm{Cl}_{2}\right): \lambda_{\max }\left(\varepsilon\left[\mathrm{M}^{-1} \mathrm{Cm}^{-1}\right]\right)=427\left(2.0 \times 10^{5}\right)$, $534\left(1.5 \times 10^{4}\right)$, and $574\left(1.1 \times 10^{4}\right)$.

18Ni: ${ }^{1} \mathrm{H}$ NMR (600 MHz, $\mathrm{CDCl}_{3}, 25^{\circ} \mathrm{C}$ ): $\delta=9.83$ (s, $1 \mathrm{H}$, meso), 9.12 (d, $J=5.0 \mathrm{~Hz}, 1 \mathrm{H}, \beta), 8.92(\mathrm{~d}, J=5.0 \mathrm{~Hz}, 1 \mathrm{H}, \beta), 8.83-8.79$ (m, $4 \mathrm{H}, \beta), 8.68$ (s, $1 \mathrm{H}, \beta), 7.89$ (m, 4H, Ar-o), 7.88 (d, $J=1.9 \mathrm{~Hz}, 2 \mathrm{H}, \mathrm{Ar}-\mathrm{o}), 7.73$ (m, 2H, Ar-p), 7.71 (t, $J=1.9 \mathrm{~Hz}, 1 \mathrm{H}$, Ar-p), 6.59-6.52 (m, 1H, allyl), 5.45 (d, $J=$ $16.9 \mathrm{~Hz}, 1 \mathrm{H}$, allyl), 5.31 (d, $J=8.7 \mathrm{~Hz}, 1 \mathrm{H}$, allyl), 4.70 (d, $J=6.0 \mathrm{~Hz}, 2 \mathrm{H}$, allyl), 1.50 (s, 18H, tert-butyl), 1.49 (s, 18H, tert-butyl), and 1.46 (s, $18 \mathrm{H}$, tert-butyl) ppm; ${ }^{13} \mathrm{C}$ NMR (151 MHz, $\left.\mathrm{CDCl}_{3}, 25{ }^{\circ} \mathrm{C}\right): \delta=149.08,148.97$, $143.45,143.18,142.88,142.69,142.56,142.38,142.15,142.10,140.37$, $140.29,140.23,137.36,132.77,132.44,132.17,132.09,131.78,131.14$, 129.19, 128.94, 128.84, 121.22, 121.16, 121.08, 120.82, 120.19, 119.15, 116.70, 101.48, 35.17, 35.14, 32.94, and 31.86 ppm; APCI-TOF-MS: $\mathrm{m} / \mathrm{z}=$ 970.5442. Calcd for $\mathrm{C}_{65} \mathrm{H}_{76} \mathrm{~N}_{4}{ }^{58} \mathrm{Ni}$ : $970.5418[\mathrm{M}]^{-}$; UV/Vis $\left(\mathrm{CH}_{2} \mathrm{Cl}_{2}\right): \lambda_{\max }$ $\left(\varepsilon\left[\mathrm{M}^{-1} \mathrm{~cm}^{-1}\right]\right)=411\left(2.4 \times 10^{5}\right)$ and $523\left(1.7 \times 10^{4}\right)$.

Synthesis of 19Ni: After 2Ni was generated as described in the synthesis of 3Ni-6Ni, CuCN $\cdot 2 \mathrm{LiCl}(0.2 \mathrm{M}$ solution in THF, $0.10 \mathrm{~mL}, 20 \mu \mathrm{mol})$, 2cyclohexen-1-one (19 $\mu \mathrm{L}, 200 \mu \mathrm{mol})$ and trimethylchlorosilane $(25 \mu \mathrm{L}, 200$ $\mu$ mol) were successively added. After the mixture was stirred for $2 \mathrm{~h}$ at room temperature, $3 \mathrm{M} \mathrm{HCl}$ was added to deprotect the resulting silyl ether. The organic layer was extracted with $\mathrm{CH}_{2} \mathrm{Cl}_{2}$, washed with brine, and dried over $\mathrm{Na}_{2} \mathrm{SO}_{4}$. Concentration followed by chromatographic purification eluting with $\mathrm{CH}_{2} \mathrm{Cl}_{2} /$ hexane afforded a solid. Recrystallization from $\mathrm{CH}_{2} \mathrm{Cl}_{2}$ /methanol gave 19Ni (70 mg, $68 \mu \mathrm{mol}, 68 \%$ ).

19Ni: ${ }^{1} \mathrm{H}$ NMR (600 MHz, $\mathrm{CDCl}_{3}, 25^{\circ} \mathrm{C}$ ): $\delta=9.76$ (s, $1 \mathrm{H}$, meso), 9.12 (d, $J=4.6 \mathrm{~Hz}, 1 \mathrm{H}, \beta$ ), 8.92 (d, $J=4.6 \mathrm{~Hz}, 1 \mathrm{H}, \beta), 8.81-8.78$ (m, 4H, $\beta$ ), 8.69 (s, $1 \mathrm{H}, \beta$ ), 7.90-7.84 (brs, 6H, Ar-o), 7.74 (t, $J=1.9 \mathrm{~Hz}, 2 \mathrm{H}, \mathrm{Ar}-p), 7.73$ (t, $J=1.9 \mathrm{~Hz}, 2 \mathrm{H}, \operatorname{Ar}-p), 7.70(\mathrm{t}, J=1.9 \mathrm{~Hz}, 1 \mathrm{H}, \operatorname{Ar}-p), 4.72(\mathrm{~m}, 1 \mathrm{H}$, cyclohexyl), $3.29(\mathrm{~m}, 1 \mathrm{H}$, cyclohexyl), $3.06(\mathrm{t}, J=12.84 \mathrm{~Hz}, 1 \mathrm{H}$, cyclohexyl), 2.78 (m, 1H, cyclohexyl), 2.69 (m, 1H, cyclohexyl), 2.64 (m, $1 \mathrm{H}$, cyclohexyl), 2.40 (m, 2H, cyclohexyl), 2.22 (m, 1H, cyclohexyl), 1.50 (s, 18H, tert-butyl), 1.49 (s, 18H, tert-butyl), and 1.46 (s, 18H, tert-butyl) ppm; ${ }^{13} \mathrm{C}$ NMR (151 MHz, $\mathrm{CDCl}_{3}, 25{ }^{\circ} \mathrm{C}$ ): $\delta=211.17,149.12,149.01$, $148.07,143.25,143.02,142.80,142.64,142.52,140.68,140.22,140.15$, 140.06, 132.60, 132.28, 132.26, 132.00, 129.24, 128.91, 128.83, 128.49, 121.29, 121.22, 121.17, 120.89, 120.28, 119.31, 100.76, 49.86, 41.70, 38.11, 35.18, 35.16, 35.13, 34.02, 31.85, 31.82, 25.87 ppm; APCI-TOFMS: $\mathrm{m} / \mathrm{z}=1026.5703$. Calcd for $\mathrm{C}_{68} \mathrm{H}_{80} \mathrm{ON}_{4}{ }^{58} \mathrm{Ni}: 1026.5680[\mathrm{M}]$; $\mathrm{UV} / \mathrm{Vis}$ $\left(\mathrm{CH}_{2} \mathrm{Cl}_{2}\right): \lambda_{\max }\left(\varepsilon\left[\mathrm{M}^{-1} \mathrm{Cm}^{-1}\right]\right)=412\left(2.6 \times 10^{5}\right)$ and $524\left(1.9 \times 10^{4}\right)$.

Synthesis of 20Ni: After 2Ni was generated as described in the synthesis of 9Ni and 10Ni, CuCN•2LiCl (0.2 M solution in THF, $0.20 \mathrm{~mL}, 40 \mu \mathrm{mol})$ and 2-naphthoyl chloride (76 mg, $400 \mu \mathrm{mol}$ ) were added. The resulting mixture was stirred for $2 \mathrm{~h}$ at room temperature and then was quenched with an $\mathrm{NH}_{4} \mathrm{Cl}$ solution The organic compounds were extracted with $\mathrm{CH}_{2} \mathrm{Cl}_{2}$, washed with brine, and dried over $\mathrm{Na}_{2} \mathrm{SO}_{4}$. After removal of the solvent in vacuo, the residue was separated by silica gel chromatography eluting with $\mathrm{CH}_{2} \mathrm{Cl}_{2} /$ hexane. Recrystallization from $\mathrm{CH}_{2} \mathrm{Cl}_{2} /$ methanol gave 20Ni (77 mg, $62 \mu \mathrm{mol}, 62 \%)$.

20Ni: ${ }^{1} \mathrm{H}$ NMR (600 MHz, $\mathrm{CDCl}_{3}, 25^{\circ} \mathrm{C}$ ): $\delta=11.11$ (s, $1 \mathrm{H}$, meso), 9.13 (s, $2 \mathrm{H}, \beta$ ), 8.81 (d, $J=5.0 \mathrm{~Hz}, 2 \mathrm{H}, \beta$ ), 8.79 (d, $J=5.0 \mathrm{~Hz}, 2 \mathrm{H}, \beta$ ), 8.68 (s, $2 \mathrm{H}$, naphthyl), 8.35 (d, $J=8.7 \mathrm{~Hz}, 2 \mathrm{H}$, naphthyl), 8.00 (d, $J=8.7 \mathrm{~Hz}, 2 \mathrm{H}$, naphthyl), 7.94 (d, $J=8.3 \mathrm{~Hz}, 2 \mathrm{H}$, naphthyl), 7.89 (d, $J=8.7 \mathrm{~Hz}, 2 \mathrm{H}$, naphthyl), 7.88 (d, $J=1.9 \mathrm{~Hz}, 4 \mathrm{H}$, Ar-o), 7.85 (d, $J=1.9 \mathrm{~Hz}, 2 \mathrm{H}$, Ar-o), $7.73(\mathrm{t}, J=1.8 \mathrm{~Hz}, 2 \mathrm{H}, \mathrm{Ar}-p), 7.64$ (t, $J=1.8 \mathrm{~Hz}, 1 \mathrm{H}, \mathrm{Ar}-p), 7.62$ (t, $J=8.3$ Hz, 2H, naphthyl), 7.53 (t, $J=8.3 \mathrm{~Hz}, 2 \mathrm{H}$, naphthyl), 1.47 (s, 18H, tert- 
butyl), and 1.42 (s, 36H, tert-butyl) ppm; ${ }^{13} \mathrm{C}$ NMR (151 MHz, $\mathrm{CDCl}_{3}$, $25^{\circ} \mathrm{C}$ ): $\delta=192.65,149.33,144.22,143.82,141.64,140.34,140.20,139.64$ 139.29, 137.35, 137.14, 135.63, 133.34, 133.23, 132.61, 132.50, 129.90, 128.83, 128.70, 128.49, 128.46, 127.98, 126.75, 126.17, 121.92, 121.67, 121.54, 121.33, 105.87, 35.16, 35.10, 31.82, and 31.79 ppm; APCI-TOFMS: $m / z=1238.5948$. Calcd for $\mathrm{C}_{84} \mathrm{H}_{84} \mathrm{O}_{2} \mathrm{~N}_{4}{ }^{58} \mathrm{Ni}: 1238.5942$ [M] ${ }^{-}$UV/Vis $\left(\mathrm{CH}_{2} \mathrm{Cl}_{2}\right): \lambda_{\max }\left(\varepsilon\left[\mathrm{M}^{-1} \mathrm{Cm}^{-1}\right]\right)=439\left(2.1 \times 10^{5}\right), 544\left(1.8 \times 10^{4}\right)$, and $580(9.5$ $\left.\times 10^{3}\right)$.

Synthesis of 22Ni-24Ni: Porphyrinyl Grignard reagent 2Ni was generated as described in the synthesis of $\mathbf{3 N i - 6 N i . ~ T o ~ t h e ~ r e s u l t i n g ~ r e d ~ s o l u t i o n , ~}$ $\mathrm{ZnCl}_{2}$ (tmeda) (38 mg, $150 \mu \mathrm{mol}$ ) was added. After stirring for $30 \mathrm{~min}$ at room temperature, $\mathrm{Pd}_{2}(\mathrm{dba})_{3}(1.5 \mathrm{mg}, 1.7 \mu \mathrm{mol})$, Ruphos (3.1 mg, 6.7 $\mu \mathrm{mol}$ ), and aryl bromide ( $83 \mu \mathrm{mol})$ were added, and then the reaction mixture was stirred for $6 \mathrm{~h}$ at $60{ }^{\circ} \mathrm{C}$. The reaction mixture was quenched with water, extracted with $\mathrm{CH}_{2} \mathrm{Cl}_{2}$, washed with brine, and dried over $\mathrm{Na}_{2} \mathrm{SO}_{4}$. After removal of the solvent in vacuo, the residue was separated by silica gel chromatography eluting with $\mathrm{CH}_{2} \mathrm{Cl}_{2} /$ hexane. Recrystallization from $\mathrm{CH}_{2} \mathrm{Cl}_{2} /$ methanol gave 22Ni-24Ni.

22Ni: ${ }^{1} \mathrm{H}$ NMR (600 MHz, $\mathrm{CDCl}_{3}, 2{ }^{\circ} \mathrm{C}$ ): $\delta=9.83$ (s, $1 \mathrm{H}$, meso), 9.05 (d, $J=4.6 \mathrm{~Hz}, 1 \mathrm{H}, \beta$ ), 8.90 (d, $J=4.6 \mathrm{~Hz}, 1 \mathrm{H}, \beta), 8.86$ (s, $1 \mathrm{H}, \beta), 8.82$ (m, 4H, $\beta$ ), 8.01 (d, $J=8.7 \mathrm{~Hz}, 2 \mathrm{H}, 4-\mathrm{OMe}-\mathrm{Ph}$ ), 7.91 (d, $J=2.0 \mathrm{~Hz}, 2 \mathrm{H}, \mathrm{Ar}-\mathrm{o}$ ), 7.89 (d, $J=1.9 \mathrm{~Hz}, 2 \mathrm{H}, \mathrm{Ar}-o$ ), 7.88 (d, $J=1.9 \mathrm{~Hz}, 2 \mathrm{H}, \mathrm{Ar}-o$ ), 7.73 (m, 2H, Ar-p), 7.71 (t, $J=1.9 \mathrm{~Hz}, 1 \mathrm{H}, \mathrm{Ar}-p$ ), 7.28 (d, $J=8.7 \mathrm{~Hz}, 2 \mathrm{H}, 4-\mathrm{OMe}-\mathrm{Ph}$ ), 4.01 (s, 3H, OMe), 1.48 (s, 36H, tert-butyl), and 1.46 (s, 18H, tert-butyl) ppm; ${ }^{13} \mathrm{C}$ NMR (151 MHz, $\left.\mathrm{CDCl}_{3}, 25{ }^{\circ} \mathrm{C}\right): \delta=159.63,149.10,149.01,145.54$, 143.26, 143.15, 143.05, 142.77, 142.71, 142.66, 141.76, 140.91, 140.28, $140.23,140.12,132.76,132.55,132.33,132.31,132.24,132.03,130.09$, 129.20, 128.98, 128.94, 128.85, 121.22, 121.21, 120.59, 120.15, 119.40, 114.65, 104.35, 55.67, 35.15, 35.14, and $31.86 \mathrm{ppm}$; APCI-TOF-MS: $\mathrm{m} / \mathrm{z}=$ 1036.5531. Calcd for $\mathrm{C}_{69} \mathrm{H}_{78} \mathrm{ON}_{4}{ }^{58} \mathrm{Ni}$ : $1036.5524[\mathrm{M}]^{-}$; UV/Vis $\left(\mathrm{CH}_{2} \mathrm{Cl}_{2}\right)$ : $\lambda_{\max }\left(\varepsilon\left[\mathrm{M}^{-1} \mathrm{~cm}^{-1}\right]\right)=415\left(2.5 \times 10^{5}\right)$ and $526\left(2.0 \times 10^{4}\right)$.

23Ni: ${ }^{1} \mathrm{H}$ NMR (600 MHz, $\mathrm{CDCl}_{3}, 2{ }^{\circ} \mathrm{C}$ ): $\delta=9.85$ (s, $1 \mathrm{H}$, meso), 9.03 (d, $J=4.6 \mathrm{~Hz}, 1 \mathrm{H}, \beta), 8.96$ (s, $1 \mathrm{H}, \beta), 8.89$ (d, $J=4.6 \mathrm{~Hz}, 1 \mathrm{H}, \beta), 8.82$ (m, 4H, $\beta$ ), 8.18 (d, $J=8.3 \mathrm{~Hz}, 2 \mathrm{H}, 4$-Bpin-Ph), 8.11 (d, $J=8.3 \mathrm{~Hz}, 2 \mathrm{H}, 4-\mathrm{Bpin}-\mathrm{Ph}$ ), 7.92 (d, $J=1.8 \mathrm{~Hz}, 2 \mathrm{H}, \mathrm{Ar}-\mathrm{o}$ ), 7.89 (d, $J=1.9 \mathrm{~Hz}, 2 \mathrm{H}, \mathrm{Ar}-\mathrm{o}$ ), 7.88 (d, $J=$ $1.8 \mathrm{~Hz}, 2 \mathrm{H}, \mathrm{Ar}-o$ ), 7.73 (m, 2H, Ar-p), 7.71 (t, $J=1.8 \mathrm{~Hz}, 1 \mathrm{H}, \mathrm{Ar}-p$ ), 1.48 (s, 36H, tert-butyl), 1.46 (s, 18H, tert-butyl), and 1.45 (s, 12H, Bpin) ppm; ${ }^{13} \mathrm{C}$ NMR $\left(151 \mathrm{MHz}, \mathrm{CDCl}_{3}, 25{ }^{\circ} \mathrm{C}\right): \delta=149.16,149.12,149.02,145.48$, $143.28,143.15,142.97,142.79,141.51,140.67,140.24,140.14,140.08$, $139.46,135.47,132.83,132.55,132.45,132.34,132.23,130.77,130.64$, $129.07,128.95,128.85,121.26,121.21,120.65,120.12,119.72,104.30$, 84.14, 35.18, 35.16, 35.14, 31.85, and 25.13 ppm; APCI-TOF-MS: $m / z=$ 1132.6261. Calcd for $\mathrm{C}_{74} \mathrm{H}_{87} \mathrm{O}_{2} \mathrm{~N}_{4}{ }^{11} \mathrm{~B}^{58} \mathrm{Ni}$ : $1132.6282 \quad[\mathrm{M}]^{-}$;UV/Vis $\left(\mathrm{CH}_{2} \mathrm{Cl}_{2}\right): \lambda_{\max }\left(\varepsilon\left[\mathrm{M}^{-1} \mathrm{~cm}^{-1}\right]\right)=416\left(2.1 \times 10^{5}\right)$ and $526\left(1.9 \times 10^{4}\right)$.

24Ni: ${ }^{1} \mathrm{H}$ NMR (600 MHz, $\left.\mathrm{CDCl}_{3}, 25^{\circ} \mathrm{C}\right): \delta=9.81$ (s, $1 \mathrm{H}$, meso), 9.03 (d, $J=4.6 \mathrm{~Hz}, 1 \mathrm{H}, \beta$ ), 8.96 (s, $1 \mathrm{H}, \beta$ ), 8.90 (d, $J=4.6 \mathrm{~Hz}, 1 \mathrm{H}, \beta), 8.82$ (m, 4H, $\beta$ ), 8.80 (s, $1 \mathrm{H}, 3-\mathrm{CO}_{2}$ TIPS-Ph), 8.31 (d, $J=7.8 \mathrm{~Hz}, 1 \mathrm{H}, 3-\mathrm{CO}_{2} \mathrm{TIPS}-\mathrm{Ph}$ ), 8.27 (d, $J=7.8 \mathrm{~Hz}, 1 \mathrm{H}, 3-\mathrm{CO}_{2}$ TIPS-Ph), 7.90 (d, $J=1.9 \mathrm{~Hz}, 2 \mathrm{H}, \mathrm{Ar}-\mathrm{o}$ ), 7.89 (d, $J=1.8 \mathrm{~Hz}, 2 \mathrm{H}, \mathrm{Ar}-\mathrm{o}$ ), 7.87 (d, $J=1.8 \mathrm{~Hz}, 2 \mathrm{H}, \mathrm{Ar}-\mathrm{o}$ ), 7.82 (t, $J=$ $7.8 \mathrm{~Hz}, 1 \mathrm{H}, 3-\mathrm{CO}_{2}$ TIPS-Ph), 7.74 (m, 2H, Ar-p), 7.71 (t, $J=1.8 \mathrm{~Hz}, 1 \mathrm{H}$, Ar-p), 1.49 (s, 18H, tert-butyl), 1.48 (s, 18H, tert-butyl), 1.46 (s, 18H, tertbutyl), 1.45 (m, $J=7.3 \mathrm{~Hz}, 3 \mathrm{H}, \mathrm{TIPS}$ ), and 1.16 (d, $J=7.3 \mathrm{~Hz}, 18 \mathrm{H}$, TIPS) ppm; ${ }^{13} \mathrm{C}$ NMR (151 MHz, $\left.\mathrm{CDCl}_{3}, 25{ }^{\circ} \mathrm{C}\right): \delta=166.47,149.14,149.05$, $144.44,143.27,143.20,143.10,142.99,142.82,142.78,141.36,140.41$, $140.17,140.04,140.03,136.98,135.58,132.69,132.64,132.60,132.97$, $132.51,132.48,132.44,132.33,130.95,129.42,129.22,128.95,128.84$, $128.80,121.41,121.30,121.25,120.73,120.12,119.75,103.95,35.17$, 31.86, 31.83, 18.09, and 12.28 ppm; APCI-TOF-MS: $m / z=1206.6636$. Calcd for $\mathrm{C}_{78} \mathrm{H}_{96} \mathrm{O}_{2} \mathrm{~N}_{4}{ }^{58} \mathrm{NiSi}$ : 1206.6651 [M]-; UV/Vis $\left(\mathrm{CH}_{2} \mathrm{Cl}_{2}\right)$ : $\lambda_{\max }\left(\varepsilon\left[\mathrm{M}^{-}\right.\right.$ $\left.\left.{ }^{1} \mathrm{~cm}^{-1}\right]\right)=415\left(2.4 \times 10^{5}\right)$ and $527\left(1.8 \times 10^{4}\right)$.

Crystal Data:
6Ni: $\mathrm{C}_{64} \mathrm{H}_{74} \mathrm{ON}_{4} \mathrm{Cl}_{2} \mathrm{Ni} ; M_{\mathrm{r}}=1044.88$; monoclinic; space group $C 2 / \mathrm{c}$ (No. 15); $a=36.906(12), b=15.540(4), c=25.945(8) \AA ; \beta=131.579(5)^{\circ} ; V=$ $11131(6) \AA^{3} ; Z=8 ; \rho_{\text {calcd }}=1.247 \mathrm{~g} / \mathrm{cm}^{3} ; T=93 \mathrm{~K} ; R_{1}=0.0564[\mathrm{I}>2 \sigma(\mathrm{I})]$; $R_{\mathrm{w}}=0.1554$ (all data); GOF $=1.043$. Crystals were grown from $\mathrm{CH}_{2} \mathrm{Cl}_{2} / \mathrm{MeOH}$.

15Ni: $\mathrm{C}_{74.51} \mathrm{H}_{89.22} \mathrm{O}_{1.19} \mathrm{~N}_{4} \mathrm{Ni} ; M_{\mathrm{r}}=1118.71$; monoclinic; space group $C$ 2/C (No. 15); $a=39.08(3), b=9.117(5), c=38.96(3) \AA ; \beta=116.07(2)^{\circ} ; V=$ $12471(15) \AA^{3} ; Z=8 ; \rho_{\text {calcd }}=1.192 \mathrm{~g} / \mathrm{cm}^{3} ; T=93 \mathrm{~K} ; R_{1}=0.1049[\mathrm{I}>2 \sigma(\mathrm{I})]$; $R_{\mathrm{w}}=0.2909$ (all data); GOF $=1.092$. Crystals were grown from toluene/ $\mathrm{MeOH}$.

20Ni: $\mathrm{C}_{87} \mathrm{H}_{84} \mathrm{O}_{2} \mathrm{~N}_{4.84} \mathrm{Cl}_{3.49} \mathrm{Ni} ; M_{\mathrm{r}}=1411.70$; triclinic, space group $P$-1 (No. 2); $a=13.491(5), b=17.043(4), c=17.188(4) \AA ; \alpha=102.5100(14), \beta=$ 92.344(9), $\gamma=106.854(8)^{\circ} ; V=3669.7(17) \AA^{3} ; Z=2 ; \rho_{\text {calcd }}=1.278 \mathrm{~g} / \mathrm{cm}^{3}$; $T=93 \mathrm{~K} ; R_{1}=0.0697[\mathrm{I}>2 \sigma(\mathrm{I})] ; R_{\mathrm{w}}=0.2269$ (all data); $\mathrm{GOF}=1.057$. Crystals were grown from $\mathrm{CHCl}_{3} / \mathrm{MeCN}$.

CCDC 991731 (6Ni), CCDC 991732 (15Ni), and CCDC 991733 (20Ni) contain the supplementary crystallographic data. These data can be obtained free of charge from the Cambridge Crystallographic Data Centre via www.ccdc.cam.ac.uk/data_request/cif.

Supporting Information (see footnote on the first page of this article): Experimental details, copies of the ${ }^{1} \mathrm{H} N M R,{ }^{13} \mathrm{C}$ NMR, and HR-MS spectra of all compounds, and X-ray crystal structure of $6 \mathrm{Ni}, 15 \mathrm{Ni}$, and $20 \mathrm{Ni}$.

[1] a) Handbook of Porphyrin Science, Vols. 1-10 (Eds: K. M. Kadish, K. M. Smith, R. Guilard), World Scientific Publishing, Singapore, 2010; b) Handbook of Porphyrin Science, Vols. 11-15 (Eds: K. M. Kadish, K. M. Smith, R. Guilard), World Scientific Publishing, Singapore, 2011; c) Handbook of Porphyrin Science, Vols. 16-25 (Eds: K. M. Kadish, K. M. Smith, R. Guilard), World Scientific Publishing, Singapore, 2012; d) The Porphyrin Handbook, Vols. 110 (Eds: K. M. Kadish, K. M. Smith, R. Guilard), Academic Press, San Diego, 2000; e) Handbook of Porphyrin Science, Vols. 11-20 (Eds: K. M. Kadish, K. M. Smith, R. Guilard), Academic Press, San Diego, 2003; f) D. Dolphin, The Porphyrins, Vols. 1 and 2, Academic Press, New York, 1979.

[2] a) F. Atefi, D. P. Arnold, J. Porphyrins Phthalocyanines 2008, 12, 801; b) T. Ren, Chem. Rev. 2008, 108, 4185; c) B. M. J. M. Suijkerbuijk, R. J. M. Klein Gebbink, Angew. Chem. 2008, 120 7506; Angew. Chem. Int. Ed. 2008, 47, 7396; d) S. Richeter, C. Jeandon, J.-P. Gisselbrecht, R. Ruppert, in Handbook of Porphyrin Science, Vol. 3 (Eds: K. M. Kadish, K. M. Smith, R. Guilard), World Scientific Publishing, Singapore, 2010, Chapter 14; e) H. Shinokubo, A. Osuka, Chem. Commun. 2009, 1011; f) S. Hiroto, S. Yamaguchi, H. Shinokubo, A. Osuka, J. Synth. Org. Chem. Jpn. 2009, 67, 688; g) H. Yorimitsu, A. Osuka, Asian J. Org. Chem. 2013, 2, 356.

[3] a) K. M. Smith, K. C. Langry, J. Chem. Soc. Chem. Commun. 1980, 217; b) K. M. Smith, K. C. Langry, J. Chem. Soc. Chem. Commun 1981, 283; c) K. M. Smith, K. C. Langry, J. Org. Chem. 1983, 48, 500; d) K. M. Smith, K. C. Langry, O. M. Minnetian, J. Org. Chem. 1984, 49, 4602; e) K. M. Smith, M. Miura, I. K. Morris, J. Org. Chem. 1986, 51, 4660; f) O. M. Minnetian, I. K. Morris, K. M. Snow, K. M. Smith, J. Org. Chem. 1989, 54, 5567; g) I. K. Morris, K. M. Snow, N. W. Smith, K. M. Smith, J. Org. Chem. 1990, 55, 1231; h) J. W. Buchler, G. Heget, Z. Naturforsch. B: Chem. Sci. 1987, 42, 1003; i) K. Sugiura, A. Kato, K. Iwasaki, H. Miyasaka, M. Yamashita, S. Hino, D. P. Arnold, Chem. Commun. 2007, 2046.

[4] a) A. G. Hyslop, M. A. Kellett, P. M. Iovine, M. J. Therien, J. Am. Chem. Soc. 1998, 120, 12676; b) N. Aratani, A. Osuka, Bull. Chem. Soc. Jpn. 2001, 74, 1361; c) H. Hata, H. Shinokubo, A. Osuka, J. Am. Chem. Soc. 2005, 127, 8264.

[5] Selected examples: a) Y. Deng, C. K. Chang, D. G. Nocera, Angew. Chem. 2000, 112, 1108; Angew. Chem. Int. Ed. 2000, 39, 1066; b) C. J. Chang, L. L. Chng, D. G. Nocera, J. Am. Chem. Soc. 2003, 125, 1866; c) T.-G. Zhang, Y. Zhao, I. Asselberghs, A. Persoons, K Clays, M. J. Therien, J. Am. Chem. Soc. 2005, 127, 9710; d) S. Yamaguchi, T. Katoh, H. Shinokubo, A. Osuka, J. Am. Chem. Soc. 2007, 129, 6392; e) N. K. S. Davis, M. Pawlicki, H. L. Anderson, 
Org. Lett. 2008, 10, 3945; f) H. Baba, J. Chen, H. Shinokubo, A Osuka, Chem. Eur. J. 2008, 14, 4256; g) I. Hisaki, S. Hiroto, K. S. Kim, S. B. Noh, D. Kim, H. Shinokubo, A. Osuka, Angew. Chem. 2007, 119, 5217; Angew. Chem. Int. Ed. 2007, 46, 5125; h) J. Song, S. Anabuki, N. Aratani, H. Shinokubo, A. Osuka, Chem. Lett. 2011, 40, 902; i) G. Bringmann, D. C. G. Götz, T. A. M. Gulder, T. H Gehrke, T. Bruhn, T. Kupfer, K. Radacki, H. Braunschweig, A Heckmann, C. Lambert, J. Am. Chem. Soc. 2008, 130, 17812; j) M. A. Filatov, R. Guilard, P. D. Harvey, Org. Lett. 2010, 12, 196; k) M. A. Bakar, N. N. Sergeeva, T. Juillard, M. O. Senge, Organometallics 2011, 30, 3225; l) K. Fujimoto, H. Yorimitsu, A. Osuka, Org. Lett. 2014, 16, 972.

[6] a) B. J. Wakefield, Organomagnesium Methods in Organic Synthesis; Academic Press, Inc.: San Diego, 1995; b) Grignard Reagents: New Developments; H. G. Richey, Ed.; Wiley, Chichester, 2000; c) The Chemistry of Organomagnesium Compounds; Z Rappoport, I. Marek, Eds.; Wiley: Chichester, 2008; d) Organometallics in Synthesis, Third Manual; M. Schlossor, Ed.; Wiley: Chichester, 2013.

[7] D.-M. Shen, C. Liu, Q.-Y. Chen, Synlett 2007, 3068.

[8] X. Jiang, D. J. Nurco, K. M. Smith, Chem. Commun. 1996, 1759; b) W. W. Kalisch, M. O. Senge, Angew. Chem. 1998, 110, 1156; Angew. Chem. Int. Ed. 1998, 37, 1107; c) M. J. Crossley, L. G. King, S. M. Pyke, C. W. Tansey, J. Porphyrins Phthalocyanines 2002, 6, 685; d) M. O. Senge, Acc. Chem. Res. 2005, 38, 733; e) M. C. Balaban, C. C.-Gillot, G. Canard, O. Fuhr, C. Roussel, T. S. Balaban, Tetrahedron 2009, 65, 3733; f) K. Yamashita, K. Kataoka, M. S. Asano, K. Sugiura, Org. Lett. 2012, 14, 190; g) S. Anabuki, S. Tokuji, N. Aratani, A. Osuka, Org. Lett. 2012, 14, 2778; h) A. A. Ryan, S. Plunkett, A. Casey, T. McCabe, M. O. Senge, Chem Commun. 2014, 50, 353; i) Q. Chen, Y.-Z. Zhu, Q.-J. Fan, S.-C Zhang, J.-Y. Zheng, Org. Lett. 2014, 16, 1590.

[9] a) K. M. Kadish, M. M. Franzen, B. C. Han, C. A.-McAdams, D. Sazou, J. Am. Chem. Soc. 1991, 113, 512; b) K. M. Kadish, M. M Franzen, B. C. Han, C. A.-McAdams, D. Sazou, Inorg. Chem. 1992, 31, 4399; c) J. Seth, D. F. Bocian, J. Am. Chem. Soc. 1994, 116, 143; d) C. J. Campbell, J. F. Rusling, C. Brückner, J. Am. Chem. Soc. 2000, 122, 6679 .
[10] a) C. E. Castro, D. Kishore, J. Organomet. Chem. 1985, 287, C27; b) K. Murakami, Y. Yamamoto, H. Yorimitsu, A. Osuka, Chem. Eur. J. 2013, 19, 9123; c) W. Zeng, M. Ishida, S. Lee, Y. M. Sung, Z. Zeng, Y. Ni, C. Chi, D. Kim, J. Wu, Chem. Eur. J. 2013, 19, 16814.

[11] a) A. Krasovskiy, P. Knochel, Angew. Chem. 2004, 116, 3396 Angew. Chem. Int. Ed. 2004, 43, 3333; b) A. Krasovskiy, B. F. Straub, P. Knochel, Angew. Chem. 2006, 118, 165; Angew. Chem. Int. Ed. 2006, 45, 159; Reviews: c) H. Ila, O. Baron, A. J. Wagner, P. Knochel, Chem. Commun. 2006, 583; d) H. Ila, O. Baron, A. J. Wagner, P. Knochel, Chem. Lett. 2006, 35, 2; e) P. Knochel, A. Krasovskiy, I. Sapountzis, in Handbook of Functionalized Organometallics; P. Knochel, Ed.; Wiley, Weinheim, 2005, Chapter 4; f) P. Knochel, A. Gavryushin, K. Brade, in The Chemistry of Organomagnesium Compounds; Z. Rappoport, I. Marek, Eds.; Wiley: Chichester, 2008, Chapter 12; g) N. M. Barl, V. Werner, C. Sämann, P. Knochel, Heterocycles 2014, 88, 827.

[12] The structures of $\mathbf{6 N i}, \mathbf{1 5 N i}$, and $\mathbf{2 0 N i}$ were confirmed by X-ray crystallographic analysis (Figures S61-63 in the Supporting Information).

[13] F. Odobel, F. Suzenet, E. Blart, J.-P. Quintard, Org. Lett. 2000, 2, 131.

[14] G. Varchi, A. Ricci, G. Cahiez, P. Knochel, Tetrahedron 2000, 56, 2727.

[15] J. E. Milne, S. L. Buchwald, J. Am. Chem. Soc. 2004, 126, 13028.

[16] P. Knochel, M. C. P. Yeh, S. C. Berk, J. Talbert, J. Org. Chem. 1988, 53, 2390.

Received: ((will be filled in by the editorial staff)) Published online: ((will be filled in by the editorial staff)) 


\section{Entry for the Table of Contents}

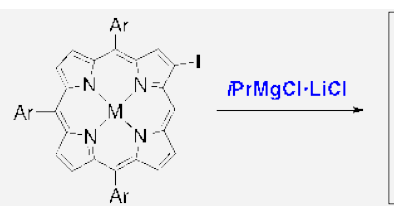

Iodine-magnesium exchange between iodoporphyrins and $i \mathrm{PrMgCl} \cdot \mathrm{LiCl}$ has realized the formation of porphyrinyl Grignard reagents for the first time. Thanks to the high reactivity, the resulting porphyrinyl Grignard reagents

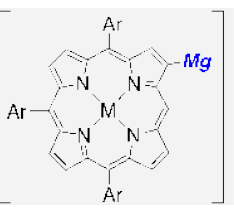

- nucleophitic addition

transmetalation to $C u$ and $Z n$

do not only react with various carbonyl compounds but also undergo transmetalation to afford porphyrinyl copper and zinc species, which participate in 1,4addition and Negishi coupling, respectively. porphyrinoids

Keisuke Fujimoto, Hideki Yorimitsu,* and Atsuhiro Osuka* Page No. - Page No.

Efficient Synthesis and Versatile Reactivity of Porphyrinyl Grignard Reagents

Keywords: porphyrin / iodinemagnesium exchange / Grignard reagent / transmetalation 